\title{
The Noether numbers and the Davenport constants of the groups of order less than 32
}

\author{
Kálmán Cziszter * ${ }^{1}$, Mátyás Domokos ${ }^{\dagger 1}$ and István Szöllősi ${ }^{\ddagger 2,1}$ \\ ${ }^{1}$ MTA Rényi Institute, 1053 Budapest, Reáltanoda utca 13-15, \\ Hungary \\ ${ }^{2}$ Faculty of Mathematics and Computer Science, Babeş-Bolyai \\ University, str. M. Kogălniceanu, nr. 1, 400084, Cluj-Napoca, \\ Romania
}

\begin{abstract}
The computation of the Noether numbers of all groups of order less than thirty-two is completed. It turns out that for these groups in nonmodular characteristic the Noether number is attained on a multiplicity free representation, it is strictly monotone on subgroups and factor groups, and it does not depend on the characteristic. Algorithms are developed and used to determine the small and large Davenport constants of these groups. For each of these groups the Noether number is greater than the small Davenport constant, whereas the first example of a group whose Noether number exceeds the large Davenport constant is found, answering partially a question posed by Geroldinger and Grynkiewicz.

2010 MSC: 13A50 (Primary) 20D60 (Secondary)

Keywords: polynomial invariant, product-one sequence, degree bound, Noether number, Davenport constant
\end{abstract}

\section{Introduction}

\subsection{The Noether number}

Fix a base field $\mathbb{F}$ and a finite group $G$. Given a $G$-module $V$ (i.e. a finite dimensional $\mathbb{F}$-vector space $V$ together with an action of $G$ via linear transformations) there is an induced action of $G$ on the symmetric tensor algebra $S(V)$ by $\mathbb{F}$-algebra automorphisms. More concretely, $S(V)$ can be identified with the polynomial algebra $\mathbb{F}\left[x_{1}, \ldots, x_{n}\right]$ where $x_{1}, \ldots, x_{n}$ is a basis of $V$, on

\footnotetext{
*Email: cziszter.kalman@gmail.com

Partially supported by National Research, Development and Innovation Office, NKFIH grants PD113138, ERC HU 15118286 and K115799.

†Email: domokos.matyas@renyi.mta.hu

Supported by National Research, Development and Innovation Office, NKFIH K 119934.

‡Email: szollosi@gmail.com

Supported by ERC-AdG 321104 and GTC-31816 (Babeş-Bolyai University grant).
} 
which $G$ acts via linear substitutions of the variables. Noether 27] proved that the algebra

$$
S(V)^{G}=\{f \in S(V): g \cdot f=f \text { for all } g \in G\}
$$

of polynomial invariants is generated by finitely many homogeneous elements. Denote by $\beta\left(S(V)^{G}\right)$ the minimal non-negative integer $d$ such that $S(V)^{G}$ is generated by its homogeneous components of degree at most $d$. Here we refer to the standard grading on $S(V)$, so the variables $x_{i}$ all have degree one. The Noether number of $G$ is

$$
\beta^{\mathbb{F}}(G)=\sup \left\{\beta\left(S(V)^{G}\right): V \text { is a } G \text {-module }\right\} .
$$

The following general facts are well known:

$$
\beta^{\mathbb{F}}(G) \begin{cases}=\infty & \text { when } \operatorname{char}(\mathbb{F})|| G \mid \\ \leq|G| & \text { when } \operatorname{char}(\mathbb{F}) \nmid|G| ;\end{cases}
$$

(see [29] for the case $\operatorname{char}(\mathbb{F})|| G \mid$, 26] for the case $\operatorname{char}(\mathbb{F})=0$ and [14, 15] for the case $0<\operatorname{char}(\mathbb{F}) \nmid|G|)$.

From now on we assume that $\mathbb{F}$ is a fixed base field with $\operatorname{char}(\mathbb{F}) \nmid|G|$, and write $\beta(G):=\beta^{\mathbb{F}}(G)$ by suppressing from the notation the dependence of the Noether number on $\mathbb{F}$. It is well known that the Noether number is unchanged when we extend the base field (see Subsection 4.3 for more information), so we may assume in proofs that $\mathbb{F}$ is algebraically closed.

The exact value of the Noether number is known only for a very limited class of groups. First of all, we have

$$
\beta(G)=\mathrm{D}(G) \text { for abelian } G
$$

where $\mathrm{D}(G)$ is the Davenport constant (the maximal length of an irreducible zero-sum sequence over $G$ ); this observation was used first in 30. The exact value of $\mathrm{D}(G)$ is known among others for abelian $p$-groups and for abelian groups of rank at most two. Considering non-abelian groups, the Noether number of the dihedral groups was determined in 30 (and in 32 for non-modular positive characteristic) along with the Noether numbers of the quaternion group of order 8 and the alternating group $A_{4}$. Recent works of the first two authors of the present paper (see [7, [8], 4, [5], [10]) added a few more (series) of groups to this short list. These results indicated that a complete table of the Noether numbers of "small" groups might be within reach. It turned out that indeed, the reduction lemmas from 7 and considerations similar to the methods used in the above mentioned papers are sufficient to determine the Noether numbers for all groups of order less than 32 . Note that the number of non-abelian groups of order 32 is 44 . This explains our choice of limiting the scope of this paper to the groups of order less than 32 .

\subsection{The Davenport constants}

Equality (2) inspired Geroldinger and Grynkiewicz [18 to look for an analogue in the case of non-abelian groups. By a sequence over the finite group $G$ we mean a finite sequence of elements from $G$ which is unordered and repetition of terms is allowed. A sequence is product-one if the product of its elements in an 
appropriate order is $1_{G}$. A sequence is product-one free if it has no product-one subsequences. The small Davenport constant $\mathrm{d}(G)$ was defined in 28 as the maximal length of a product-one free sequence. A sequence is considered as an element of the free abelian monoid $\mathcal{F}(G)$ and product-one sequences form a submonoid $\mathcal{B}(G)$ of $\mathcal{F}(G)$. The large Davenport constant $\mathrm{D}(G)$ was defined in [18] as the maximal length of an atom (irreducible element) in $\mathcal{B}(G)$. We have the inequality

$$
\mathrm{d}(G)+1 \leq \mathrm{D}(G)
$$

with equaliy for abelian $G$. The question whether $\beta(G)$ is always between $\mathrm{d}(G)+$ 1 and $\mathrm{D}(G)$ was raised in [18] (the possible relation between the Noether number and Davenport constants is discussed further in [10]). Using the implementation of our algorithms presented in Section 6 we completed the determination of $\mathrm{d}(G)$ and $\mathrm{D}(G)$ for groups of order less than 32. It turned out that $\mathrm{d}(G)+1 \leq \beta(G) \leq$ $\mathrm{D}(G)$ holds for these groups with the only exception being the Heisenberg group $H_{27}$ of order 27 , for which we have $\beta\left(H_{27}\right)>\mathrm{D}\left(H_{27}\right)$.

\subsection{Outline of the paper}

In Section 2 we give a table containing the values of the Noether number and the Davenport constants for each non-abelian group of order less than 32. In Section 3 we provide references and proofs to verify the Noether numbers in the table. We draw consequences from the obtained data and state some open questions in Section 4. In particular, in non-modular characteristic for each group of order less than 32 the Noether number is attained on a multiplicity free representation (see Theorem 4.2), the Noether number is strictly monotone with respect to taking subgroups or factor groups (see Theorem 4.5, which is generalized to arbitrary finite groups in the subsequent paper 9]) and the Noether number does not depend on the characteristic (see Theorem 4.7). In Section 5 we turn to the Davenport constants. Notation and known results are recalled in Sections 5.1 and 5.2. In Section 5.3 we present a theoretical proof for the fact that $\mathrm{D}\left(H_{27}\right)=8$; this seems to be of special interest because so far this is the only known example of a group for which the Noether number is greater than the large Davenport constant. Section 6 contains the description of the algorithms we employed to compute the Davenport constants given in the table in Section 2

\section{Noether numbers and Davenport constants}

The classification of all groups of order less than 32 is given e.g. in 24, Chapter 22]. In the table below we present the Noether numbers and Davenport constants of all the non-abelian groups of order less than 32. In the first column we also give for reference the GAP (see [16]) identification numbers $(m, n)$ using which these groups can be constructed in GAP by the function call SmallGroup $(m, n)$. 


\begin{tabular}{|c|c|c|c|c|c|}
\hline GAP & $G$ & d & $\beta$ & $\mathrm{D}$ & reference for $\beta$ \\
\hline$(6,1)$ & $S_{3}=D i h_{6}$ & 3 & 4 & 6 & {$[8$, Theorem 10.3] } \\
\hline$(8,3)$ & $D_{i h_{8}}$ & 4 & 5 & 6 & [8, Theorem 10.3] \\
\hline$(8,4)$ & $Q_{8}=D i c_{8}$ & 4 & 6 & 6 & [8, Theorem 10.3] \\
\hline$(10,1)$ & $D i h_{10}$ & 5 & 6 & 10 & [8, Theorem 10.3] \\
\hline$(12,1)$ & $D i c_{12}=C_{3} \rtimes C_{4}$ & 6 & 8 & 9 & [8, Theorem 10.3] \\
\hline$(12,3)$ & $A_{4}$ & 4 & 6 & 7 & [7. Theorem 3.4] \\
\hline$(12,4)$ & $\operatorname{Dih}_{12}$ & 6 & 7 & 9 & [8, Theorem 10.3] \\
\hline$(14,1)$ & $\operatorname{Dih}_{14}$ & 7 & 8 & 14 & [8, Theorem 10.3] \\
\hline$(16,3)$ & $\left(C_{2} \times C_{2}\right) \rtimes C_{4}=\left(C_{4} \times C_{2}\right) \rtimes_{\psi} C_{2}$ & 5 & 6 & 7 & Proposition 3.10 \\
\hline$(16,4)$ & $C_{4} \rtimes C_{4}$ & 6 & 7 & 8 & Proposition 3.1 \\
\hline$(16,6)$ & $M_{16}$ & 8 & 9 & 10 & [8, Theorem 10.3] \\
\hline$(16,7)$ & $\operatorname{Dih}_{16}$ & 8 & 9 & 12 & [8, Theorem 10.3] \\
\hline$(16,8)$ & $S D_{16}$ & 8 & 9 & 12 & [8, Theorem 10.3] \\
\hline$(16,9)$ & Dic $_{16}$ & 8 & 10 & 12 & [8, Theorem 10.3] \\
\hline$(16,11)$ & $D_{i h_{8}} \times C_{2}=\left(C_{4} \times C_{2}\right) \rtimes_{-1} C_{2}$ & 5 & 6 & 7 & [8, Corollary 5.5] \\
\hline$(16,12)$ & $Q_{8} \times C_{2}$ & 5 & 7 & 7 & Proposition 3.2 \\
\hline$(16,13)$ & $($ Pauli $)=\left(C_{4} \times C_{2}\right) \rtimes_{\phi} C_{2}$ & 5 & 7 & 7 & [10. Example 5.4] \\
\hline$(18,1)$ & $\operatorname{Dih}_{18}$ & 9 & 10 & 18 & [8, Theorem 10.3] \\
\hline$(18,3)$ & $S_{3} \times C_{3}$ & 7 & 8 & 10 & Proposition 3.3 \\
\hline$(18,4)$ & $\left(C_{3} \times C_{3}\right) \rtimes_{-1} C_{2}$ & 5 & 6 & 10 & [8, Corollary 5.5] \\
\hline$(20,1)$ & $D i c_{20}$ & 10 & 12 & 15 & [8, Theorem 10.3] \\
\hline$(20,3)$ & $C_{5} \rtimes C_{4}$ & 7 & 8 & 10 & [7, Proposition 3.2] \\
\hline$(20,4)$ & $D i h_{20}$ & 10 & 11 & 15 & [8, Theorem 10.3] \\
\hline$(21,1)$ & $C_{7} \rtimes C_{3}$ & 8 & 9 & 14 & [7. Proposition 2.24] \\
\hline$(22,1)$ & $\operatorname{Dih}_{22}$ & 11 & 12 & 22 & [8, Theorem 10.3] \\
\hline$(24,1)$ & $C_{3} \rtimes C_{8}$ & 12 & 13 & 15 & [8, Theorem 10.3] \\
\hline$(24,3)$ & $S L_{2}\left(\mathbb{F}_{3}\right)=\tilde{A}_{4}$ & 7 & 12 & 13 & [7, Corollary 3.6] \\
\hline$(24,4)$ & $D i c_{24}=C_{3} \rtimes Q_{8}$ & 12 & 14 & 18 & [8, Theorem 10.3] \\
\hline$(24,5)$ & $\operatorname{Dih}_{6} \times C_{4}$ & 12 & 13 & 15 & [8, Theorem 10.3] \\
\hline$(24,6)$ & $D i h_{24}$ & 12 & 13 & 18 & [8, Theorem 10.3] \\
\hline$(24,7)$ & $D i c_{12} \times C_{2}$ & 8 & 9 & 11 & Proposition 3.4 \\
\hline$(24,8)$ & $C_{3} \rtimes \operatorname{Dih}_{8}=\left(C_{6} \times C_{2}\right) \rtimes_{\gamma} C_{2}$ & 7 & 9 & 14 & Proposition 3.5 \\
\hline$(24,10)$ & $D i h_{8} \times C_{3}$ & 12 & 13 & 14 & [8, Theorem 10.3] \\
\hline$(24,11)$ & $Q_{8} \times C_{3}$ & 12 & 13 & 14 & [8, Theorem 10.3] \\
\hline$(24,12)$ & $S_{4}$ & 6 & 9 & 12 & [10, Example 5.3] \\
\hline$(24,13)$ & $A_{4} \times C_{2}$ & 7 & 8 & 10 & Proposition 3.8 \\
\hline$(24,14)$ & $D i h_{12} \times C_{2}=\left(C_{6} \times C_{2}\right) \rtimes_{-1} C_{2}$ & 7 & 8 & 10 & [8, Corollary 5.5] \\
\hline$(26,1)$ & $\operatorname{Dih}_{26}$ & 13 & 14 & 26 & [8, Theorem 10.3] \\
\hline$(27,3)$ & $H_{27}=U T_{3}\left(\mathbb{F}_{3}\right)$ & 6 & 9 & 8 & [5, Corollary 15] \\
\hline$(27,4)$ & $M_{27}=C_{9} \rtimes C_{3}$ & 10 & 11 & 12 & [8, Remark 10.4] \\
\hline$(28,1)$ & $D i c_{28}=C_{7} \rtimes C_{4}$ & 14 & 16 & 21 & [8, Theorem 10.3] \\
\hline$(28,3)$ & $D i h_{28}$ & 14 & 15 & 21 & [8, Theorem 10.3] \\
\hline$(30,1)$ & $\operatorname{Dih}_{6} \times C_{5}$ & 15 & 16 & 18 & [8, Theorem 10.3] \\
\hline$(30,2)$ & $D i h_{10} \times C_{3}$ & 15 & 16 & 20 & [8, Theorem 10.3] \\
\hline$(30,3)$ & $D_{i h_{30}}$ & 15 & 16 & 30 & [8, Theorem 10.3] \\
\hline
\end{tabular}

In this table $S_{3}$ and $S_{4}$ are the symmetric groups of degree 3 and $4, Q_{8}$ is the quaternion group of order $8, A_{4}$ is the alternating group of degree 4 and $\tilde{A}_{4}$ 
is the binary tetrahedral group, $H_{27}$ is the Heisenberg group of order 27 (i.e. the group of unitriangular $3 \times 3$ matrices over the 3-element field). For $m \geq 2$, $D i h_{2 m}$ is the dihedral group of order $2 m, D i c_{4 m}$ is the dicyclic group given by generators and relations

$$
\text { Dic }_{4 m}:=\left\langle a, b \mid a^{2 m}=1, \quad b^{2}=a^{m}, \quad b a b^{-1}=a^{-1}\right\rangle .
$$

Using for the semidirect product of two cyclic groups the notation

$C_{m} \rtimes_{d} C_{n}=\left\langle a, b \mid a^{m}=1, b^{n}=1, b a b^{-1}=a^{d}\right\rangle \quad$ where $d \in \mathbb{N}$ is coprime to $m$ we have that $S D_{2^{k}}$ is the semidihedral group

$$
S D_{2^{k}}=C_{2^{k-1}} \rtimes_{d} C_{2}, \quad d=2^{k-2}-1 \quad(k \geq 4),
$$

and for a prime $p$ and $k \geq 3$,

$$
M_{p^{k}}=C_{p^{k-1}} \rtimes_{d} C_{p}, \quad d=p^{k-2}+1 .
$$

Moreover, the symbol $\rtimes$ always stands for a semidirect product that is not a direct product.

\section{Noether numbers}

\subsection{Abelian groups}

It has been long known that for an abelian group $G$ we have $\mathrm{d}(G)+1=\beta(G)=$ $\mathrm{D}(G)$, see [10] for a recent survey largely motivated by this fact. Therefore we can restate known results on the Davenport constants of abelian groups in terms of the Noether number:

- if $G$ is cyclic then $\beta(G)=|G|$ (see for example [30]);

- if $G$ is of rank two, i.e. $G=C_{n} \times C_{m}$ for some $m \mid n$ then $\beta(G)=n+m-1$ (see for example [19, Theorem 5.8.3]);

- if $G$ is a $p$-group, i.e. $G=C_{p^{n_{1}}} \times \ldots \times C_{p^{n_{r}}}$ then $\beta(G)=1+\sum_{i=1}^{r}\left(p^{n_{i}}-1\right)$ (see for example [19, Theorem 5.5.9]);

- if $G=C_{2} \times C_{2} \times C_{2 n}$ then $\mathrm{D}(G)=2 n+2$ by [13].

All abelian groups of order less than 32 fall under one of the four cases above. We note that more recent progress on the Davenport constants of abelian groups can be found in [1, 17, Corollary 4.2.13], [31, [2.

\subsection{Groups with a cyclic subgroup of index two}

Let $G$ be a non-cyclic group having a cyclic subgroup of index two. According to 8 , Theorem 10.3] we have

$$
\beta(G)=\frac{1}{2}|G|+ \begin{cases}2 & \text { if } G=\text { Dic }_{4 m}, m>1 \\ 1 & \text { otherwise }\end{cases}
$$

Formula (3) yields the Noether number for 27 groups out of the 45 groups from the table in Section 2 


\subsection{The generalized dihedral groups}

The semi-direct product $\operatorname{Dih}(A):=A \rtimes_{-1} C_{2}$, where $A$ is an abelian group on which $C_{2}$ acts by inversion, is called the generalized dihedral group obtained from $A$. According to [8, Corollary 5.5] we have

$$
\beta(\operatorname{Dih}(A))=\mathrm{D}(A)+1 .
$$

Combining this with the known values of Davenport constants given in Section 3.1 we can compute the Noether number of the three generalized dihedral groups of order less than 32 which are not themselves dihedral groups (these are $\left.\left(C_{4} \times C_{2}\right) \rtimes_{-1} C_{2}=D i h_{8} \times C_{2},\left(C_{3} \times C_{3}\right) \rtimes_{-1} C_{2},\left(C_{6} \times C_{2}\right) \rtimes_{-1} C_{2}=D i h_{12} \times C_{2}\right)$.

\subsection{Cases when the reduction lemmas give exact results}

The $k$ th Noether number $\beta_{k}\left(S(V)^{G}\right.$ ) (where $k$ is a positive integer) was defined in [7. Section 1.2] as the top degree of the factor space $S(V)^{G} /\left(S(V)_{+}^{G}\right)^{k+1}$, where $S(V)_{+}^{G}$ stands for the sum of the positive degree homogeneous components of $S(V)^{G}$. The supremum of $\beta_{k}(S(V))^{G}$ as $V$ ranges over all $G$-modules over $\mathbb{F}$ is denoted by $\beta_{k}(G)$ (for an abelian group $G, \beta_{k}(G)$ equals the $k$ th Davenport constant $\mathrm{D}_{k}(G)$ introduced in [22]). In the special case $k=1$ we have $\beta_{1}\left(S(V)^{G}\right)=\beta\left(S(V)^{G}\right)$ and hence $\beta_{1}(G)=\beta(G)$. Using the $k$ th Noether number one can get upper bounds on $\beta(G)$ by the following reduction lemma:

$$
\beta(G) \leq \beta_{\beta(G / N)}(N) \quad \text { for } N \triangleleft G \text { by [7, Lemma 1.4]. }
$$

Lower bounds on $\beta(G)$ can be derived from the following inequality:

$$
\beta(G) \geq \beta(G / N)+\beta(N)-1 \quad \text { if } G / N \text { is abelian by [8, Theorem 4.3]. }
$$

These results already suffice to establish the precise value of the Noether number for several groups considered below when we combine them with the following formula for the $k$ th Noether number:

$$
\beta_{k}\left(C_{n} \times C_{m}\right)=n k+m-1 \text { for } m \mid n \text { by [22, Proposition 5]. }
$$

Proposition 3.1. $\beta\left(C_{4} \rtimes C_{4}\right)=7$.

Proof. We have the lower bound $\beta\left(C_{4} \rtimes C_{4}\right) \geq 2 \beta\left(C_{4}\right)-1=7$ by (6). On the other hand $G=C_{4} \rtimes C_{4}$ contains a normal subgroup isomorphic to the Klein four-group $K_{4}=C_{2} \times C_{2}$ such that $G / K_{4} \cong K_{4}$. Hence by (5) and (7) we have $\beta(G) \leq \beta_{\beta\left(K_{4}\right)}\left(K_{4}\right)=7$.

Proposition 3.2. $\beta\left(Q_{8} \times C_{2}\right)=7$.

Proof. We have the lower bound $\beta\left(Q_{8} \times C_{2}\right) \geq \beta\left(Q_{8}\right)+\beta\left(C_{2}\right)-1=7$ by (6) since $\beta\left(Q_{8}\right)=6$ by [30, Lemma 10.1]. On the other hand $G$ has a normal subgroup $K_{4}$ such that $G / K_{4} \cong K_{4}$. Hence again $\beta(G) \leq \beta_{\beta\left(K_{4}\right)}\left(K_{4}\right)=7$ by (5) and (7).

Proposition 3.3. $\beta\left(S_{3} \times C_{3}\right)=8$.

Proof. We have $\beta\left(S_{3} \times C_{3}\right)=\beta\left(C_{3} \rtimes_{-1} C_{6}\right) \geq \beta\left(C_{3}\right)+\beta\left(C_{6}\right)-1=8$ by (6) and the upper bound $\beta\left(S_{3} \times C_{3}\right) \leq \beta_{2}\left(C_{3} \times C_{3}\right)=8$ by (5) and (77). 
Proposition 3.4. $\beta\left(D i c_{12} \times C_{2}\right)=9$.

Proof. We have $\beta\left(\right.$ Dic $\left._{12} \times C_{2}\right) \geq \beta\left(\right.$ Dic $\left._{12}\right)+\beta\left(C_{2}\right)-1=8+2-1=9$ by (6) and (3). On the other hand the center of the group $G=D i c_{12} \times C_{2}$ is isomorphic to the Klein four-group $K_{4}=C_{2} \times C_{2}$ and we have $G / K_{4} \cong D i h_{6}$. So we get $\beta(G) \leq \beta_{\beta\left(D i h_{6}\right)}\left(K_{4}\right)=\beta_{4}\left(K_{4}\right)=2 \cdot 4+1=9$ by (3), (5) and (7).

Proposition 3.5. $\beta\left(C_{3} \rtimes D i h_{8}\right)=9$.

Proof. The group $G$ has an index two subgroup $N \cong C_{3} \rtimes C_{4}=D i c_{12}$. By (3) we have $\beta(N)=\frac{1}{2}|N|+2=8$, implying by (6) that $\beta(G) \geq 8+2-1=9$.

For the reverse inequality observe that the kernel of the action of $D i h_{8}$ on $C_{3}$ is isomorphic to the Klein group $K_{4}=C_{2} \times C_{2}$. So the subgroup $K_{4} \leq D i h_{8}$ is normal in $G$ and as $D i h_{8} / K_{4} \cong C_{2}$ acts by inversion on $C_{3}$ we have $G / K_{4} \cong$ $D i h_{6}$. Therefore $\beta(G) \leq \beta_{\beta\left(D i h_{6}\right)}\left(K_{4}\right)=\beta_{4}\left(K_{4}\right)=2 \cdot 4+1=9$ by (3), (5) and (7).

Example 3.6. For later reference let us construct here a representation of $G=C_{3} \rtimes D_{i h_{8}}$ (where the kernel of the conjugation action of $D i h_{8}$ on $C_{3}$ is isomorphic to $C_{2} \times C_{2}$ ) on which the Noether number is attained. Consider the two-dimensional $G$-module $U=\mathbb{F}^{2}$ on which the representation is given by the matrices

$$
a=\left(\begin{array}{ll}
0 & 1 \\
1 & 0
\end{array}\right), \quad b=\left(\begin{array}{cc}
-1 & 0 \\
0 & 1
\end{array}\right), \quad c=\left(\begin{array}{cc}
\zeta & 0 \\
0 & \zeta^{2}
\end{array}\right)
$$

where $\zeta$ is a primitive third root of unity. Here $\langle a, b\rangle \cong D i h_{8}, c^{a}=c^{-1}$ and $c^{b}=$ $c$, so these matrices are indeed generating the group in question. Since this group is generated also by the pseudo-reflections $a, b$, and $a c$, the $G$-module structure of $S(U)$ is well known by the Shephard-Todd-Chevalley theorem [3], 33. In particular, $S(U)^{G}=\mathbb{F}[x, y]^{G}$ is a polynomial ring generated by $(x y)^{2}, x^{6}+$ $y^{6}$. The element $x^{7} y-x y^{7}$ is not contained in the ideal of $S(U)$ generated by the above two invariants. It spans a one-dimensional $G$-invariant subspace isomorphic to the one-dimensional $G$-module $\mathbb{F}_{\chi}$ corresponding to the order two homomorphism $\chi: G \rightarrow \mathbb{F}^{\times}$given by the determinant on $G L(U)$. It follows that in $S\left(U \oplus \mathbb{F}_{\chi}\right)$ the element $\left(x^{7} y-x y^{7}\right) z$ is an indecomposable $G$-invariant, where $z$ spans the summand $\mathbb{F}_{\chi}$.

\subsection{Some groups with an abelian normal subgroup of in- dex two or three}

In this section we shall discuss a group $G$ with an abelian normal subgroup $A$ of index 2 or 3 . Let $V$ be a $G$-module over an algebraically closed base field $\mathbb{F}$. We shall fix the following notation: $I:=S(V)^{A}, R:=S(V)^{G}$. By Clifford theory we know that each irreducible $G$-module is 1-dimensional or is induced from a 1-dimensional $A$-module. Therefore it is possible to choose the variables in $S(V)$ to be $A$-eigenvectors that are permuted up to scalars by the action of $G$. We shall tacitly assume that the variables were chosen that way. It follows that $I$ is spanned by $A$-invariant monomials, permuted by $G$ up to scalars. It will be convenient to use the notation $f^{g}=g^{-1} \cdot f$ for $g \in G$ and $f \in S(V)$. For a non-zero scalar multiple $x$ of a variable let us denote by $\theta(x) \in \widehat{A}$ its weight (i.e. $x^{a}=(\theta(x)(a)) x \in \mathbb{F} x$ for all $a \in A$ ). Here $\widehat{A}$ is the character group of $A$, i.e. the abelian group consisting of the 
group homomorphisms $A \rightarrow \mathbb{F}^{\times}$. Note that $\widehat{A} \cong A$ when $\mathbb{F}$ is algebraically closed. For a non-zero scalar multiple $w$ of a monomial $y_{1} \ldots y_{d}$ the multiset $\Phi(w)=\left\{\theta\left(y_{1}\right), \ldots, \theta\left(y_{d}\right)\right\}$ is called the weight sequence of $w$. It is a sequence over the abelian group $\widehat{A}$ (where the order of the elements in a sequence is disregarded and repetition is allowed). Sequences over $\widehat{A}$ form a monoid with multiplication denoted by "." (see Section[5]for more discussion of this monoid), such that for monomials $w, w^{\prime}$ we have $\Phi\left(w w^{\prime}\right)=\Phi(w) \cdot \Phi\left(w^{\prime}\right)$. The action of $G$ on $A$ by conjugation induces an action on $\widehat{A}$ by $\theta^{g}(a)=\theta\left(g_{a g}{ }^{-1}\right)$. For a variable $x, g \in G$ and $a \in A$ we have that $x^{g}$ is a non-zero scalar multiple of a variable, and $\left(x^{g}\right)^{a}=\left(x^{g a g^{-1}}\right)^{g}=\left(\theta(x)\left(g a g^{-1}\right) x\right)^{g}=\theta(x)^{g}(a) x^{g}$, showing the equality $\theta\left(x^{g}\right)=\theta(x)^{g}$. Consequently, for a non-zero scalar multiple $w$ of a monomial we have $\Phi\left(w^{g}\right)=\Phi(w)^{g}$ where the action of $G$ on $\widehat{A}$ is extended componentwise to sequences over $\widehat{A}$. Denote by $\tau=\tau_{A}^{G}: I \rightarrow R$ the relative transfer map $f \mapsto \sum_{g \in G / A} f^{g}$. It is a surjective $R$-module homomorphism, preserving the grading (inherited from the standard grading on the polynomial ring $S(V)$ ). The following lemma provides the common basis for the proofs of the upper bounds for Noether numbers below:

Lemma 3.7. Suppose that for any zero-sum sequence $S$ over $\widehat{A}$ with $|S|>d$ which does not factor as the product of $1+[G: A]$ non-empty zero-sum sequences, we have a factorization $S=S_{1} \cdot S_{2}$ as the product of two zero-sum sequences satisfying the following: for each $g \in G \backslash A$, the sequence $S_{1} \cdot S_{2}^{g}$ factors as the product of $1+[G: A]$ non-empty zero-sum sequences. Then we have the inequality $\beta(G) \leq d$.

Proof. By Proposition 1.5 in [7, in order to prove $\beta\left(S(V)^{G}\right) \leq d$ it is sufficient to show that $I_{>d+1} \subseteq I_{+} R_{+}$where $I_{\geq d+1}$ stands for the sum of homogeneous components of $I$ of degree at least $d+1$. That is, we need to show that any $A$ invariant monomial $w$ with $\operatorname{deg}(w)>d$ belongs to $I_{+} R_{+}$. So take an arbitrary $A$-invariant monomial with $\operatorname{deg}(w)>d$, and denote by $S$ its weight sequence $\Phi(w)$. If $S$ factors into a product of $1+[G: A]$ zero-sum sequences, then $w$ factors as the product of $1+[G: A]$ non-trivial $A$-invariant monomials. Thus $w \in\left(I_{+}\right)^{1+[G: A]}$. On the other hand by Proposition 1.6 in [7] we have $\left(I_{+}\right)^{1+[G: A]} \subseteq I_{+} R_{+}$, implying in turn that $w \in I_{+} R_{+}$. Next, suppose that $S$ does not factor as the product of $1+[G: A]$ non-empty zero-sum sequences. Then by assumption we have a factorization $S=S_{1} \cdot S_{2}$ with the properties in the statement. The monomial $w$ factorizes as $w=w_{1} w_{2}$ with $\Phi\left(w_{1}\right)=S_{1}$, $\Phi\left(w_{2}\right)=S_{2}$. We have the equality

$$
w=w_{1} \tau\left(w_{2}\right)-\sum w_{1} w_{2}^{g},
$$

where the summation above ranges over a set of representatives of the $A$-cosets in $G$ which are different from $A$. For each such summand, $\Phi\left(w_{1} w_{2}^{g}\right)=S_{1} \cdot S_{2}^{g}$ factors as the product of $1+[G: A]$ non-empty zero-sum sequences, so $w_{1} w_{2}^{g} \in$ $\left(I_{+}\right)^{1+[G: A]} \subseteq I_{+} R_{+}$. The first summand $w_{1} \tau\left(w_{2}\right)$ above is also contained in $I_{+} R_{+}$since $\tau\left(w_{2}\right) \in R_{+}$, implying in turn that $w \in I_{+} R_{+}$. Thus we showed $I_{\geq d+1} \subseteq I_{+} R_{+}$.

Proposition 3.8. $\beta\left(A_{4} \times C_{2}\right)=8$.

Proof. First we show the inequality $\beta\left(A_{4} \times C_{2}\right) \leq 8$. We have $A_{4}=K \rtimes\langle g\rangle$ where $K \cong C_{2} \times C_{2}$ and $\langle g\rangle \cong C_{3}$, so $G:=A_{4} \times C_{2}$ contains an abelian normal subgroup 
$A=K \times C_{2}$, where $g$ centralizes the last summand $C_{2}$, and conjugation by $g$ gives an order 3 automorphism of $K$. Write the character group $\widehat{A}$ additively, and denote its elements by $\{(0, \varepsilon),(a, \varepsilon),(b, \varepsilon),(c, \varepsilon) \mid \varepsilon=0,1\}$, where $(0,0)$ is the zero element, $\{(a, 0),(b, 0),(c, 0),(0,0)\}$ is a subgroup, and the action of $\langle g\rangle$ on $A$ induces the action on $\widehat{A}$ given by $(a, \varepsilon)^{g}=(b, \varepsilon),(b, \varepsilon)^{g}=(c, \varepsilon)$, $(c, \varepsilon)^{g}=(a, \varepsilon)$. We shall apply Lemma 3.7 so take a zero-sum sequence $S$ over $\widehat{A}$ with $|S| \geq 9$, such that $S$ does not factor as the product of four non-empty zero-sum sequences over $\widehat{A}$. Since $\beta_{2}(A)=7$ by Lemma 3.7 in [11, 0 does not occur in $S$ (otherwise $S=\{0\} \cdot T$ and $|T| \geq 8>\beta_{2}(A)=7$ implies that $T$ is the product of three non-empty zero-sum sequences, a contradiction). On the other hand, $|S|>7=|\widehat{A} \backslash\{(0,0)\}|$ implies that $S$ contains an element $s$ with multiplicity at least 2 , hence $S=T_{0} \cdot T$, where $T_{0}, T$ are zero-sum sequences, $T_{0}=\{s, s\}$ has length 2 , so $|T| \geq 7$. Note that $T$ does not contain a zero-sum subsequence of length 2 (since otherwise $\mathrm{D}(A)=4$ would imply that $S$ is the product of four non-empty zero-sum sequences). It follows that $T$ consists of the non-zero elements in $\widehat{A}$ (each having multiplicity one), and so $T=T_{1} T_{2}$ where $T_{1}=\{(a, 1),(b, 0),(c, 0),(0,1)\}, T_{2}=\{(a, 0),(b, 1),(c, 1)\}$. Set $S_{1}:=T_{0} \cdot T_{1}$ and $S_{2}:=T_{2}$. The factorization $S=S_{1} \cdot S_{2}$ fulfills the requirements of Lemma 3.7? indeed, $(a, 1)=(c, 1)^{g}$ occurs both in $S_{1}$ and $S_{2}^{g}$, so $S$ can be written as $S=T_{0} \cdot\{(a, 1),(a, 1)\} \cdot U$ where $U$ is a zero-sum sequence of length 5 . Hence by $\mathrm{D}(A)=4$ we get that $S_{1} \cdot S_{2}^{g}$ factors as the product of four nonempty zero-sum sequences. Similarly, $(a, 1)=(b, 1)^{g^{2}}$ occurs also in $S_{2}^{g^{2}}$, hence $S_{1} \cdot S_{2}^{g^{2}}$ also factors as the product of four non-empty zero-sum sequences. By Lemma 3.7 we conclude that $\beta(G) \leq 8$.

The subgroup $K$ is normal in $G$ and $G / K \cong C_{6}$, hence by (6) we obtain the reverse inequality $\beta(G) \geq \beta(K)+\beta\left(C_{6}\right)-1=3+6-1=8$.

Example 3.9. For later use we present a concrete $A_{4} \times C_{2}$-module on which the Noether number is attained. We keep the notation used in the proof of Proposition 3.8 Let $W$ be the 3-dimensional irreducible $A_{4}$-module (the non-trivial direct summand in the 4-dimensional standard permutation representation of $\left.A_{4}\right)$ viewed as a $G$-module under the natural surjection $G \rightarrow A_{4}$ with kernel $C_{2}$. Let $U$ be the non-trivial 1-dimensional $C_{2}$-module viewed as a $G$-module under the natural surjection $G \rightarrow C_{2}$ with kernel $A_{4}$. Set $V:=W \oplus(W \otimes U) \oplus U$. Then $S(V)=\mathbb{F}\left[x_{1}, x_{2}, x_{3}, y_{1}, y_{2}, y_{3}, z\right]$ where $x_{1}, x_{2}, x_{3}$ are $A$-eigenvectors with weight $(a, 0),(b, 0),(c, 0)$ and they are permuted cyclically by $g$. Similarly $y_{1}, y_{2}, y_{3}$ are $A$-eigenvectors with weight $(a, 1),(b, 1),(c, 1)$ and they are permuted cyclically by $g$. The last variable $z$ is fixed by $g$ and is an $A$-eigenvector with weight $(0,1)$. Denote by $\tau=\tau_{A}^{G}: I \rightarrow R$ the relative transfer map $f \mapsto f+f^{g}+f^{g^{2}}$. Then $R$ is spanned as an $\mathbb{F}$-vector space by $\tau(w)$ where $w$ ranges over the $A$-invariant monomials. There is an $\mathbb{N}^{3}$-grading on $\mathbb{F}[V]$ given by

$$
\operatorname{deg}_{3}\left(x_{1}^{a_{1}} x_{2}^{a_{2}} x_{3}^{a_{3}} y_{1}^{b_{1}} y_{2}^{b_{2}} y_{3}^{b_{3}} z^{d}\right):=\left(a_{1}+a_{2}+a_{3}, b_{1}+b_{2}+b_{3}, d\right) .
$$

This is preserved by the action of $G$, hence $I$ and $R$ are $\mathbb{N}^{3}$-graded subalgebras. Moreover, $\tau$ preserves the $\mathbb{N}^{3}$-grading. We claim that $\tau\left(x_{1}^{3} x_{2}^{3} y_{3} z\right)$ is indecomposable, that is, it is not contained in $\left(R_{+}\right)^{2}$. Suppose to the contrary that $\tau\left(x_{1}^{3} x_{2}^{3} y_{3} z\right)$ is a linear combination of elements of the form $\tau(w) \tau\left(w^{\prime}\right)$ where $w$ and $w^{\prime}$ are non-trivial $A$-invariant monomials and $\operatorname{deg}_{3}(w)+\operatorname{deg}_{3}\left(w^{\prime}\right)=(6,1,1)$. 
There is no $A$-invariant variable in $\mathbb{F}[V]$, so both $w$ and $w^{\prime}$ above have total degree at least 2 . The $\langle g\rangle$-orbits of the $A$-invariant monomials $w$ with $\operatorname{deg}_{3}(w)=(*, *, 1), \operatorname{deg}_{3}(w) \neq(6,1,1)$ and where $\operatorname{deg}_{3}(w)$ is dominated by $(6,1,1)$ are

$$
x_{1} x_{2} y_{3} z, \quad x_{1}^{3} x_{2} y_{3} z, \quad x_{1} x_{2}^{3} y_{3} z, \quad x_{1} x_{2} x_{3}^{2} y_{3} z .
$$

The $G$-invariants of degree 2 or 4 depending only on $x_{1}, x_{2}, x_{3}$ are

$\tau\left(x_{1}^{2}\right)=x_{1}^{2}+x_{2}^{2}+x_{3}^{2}, \quad \tau\left(x_{1}^{2} x_{2}^{2}\right)=x_{1}^{2} x_{2}^{2}+x_{1}^{2} x_{3}^{2}+x_{2}^{2} x_{3}^{2}, \quad \tau\left(x_{1}^{4}\right)=x_{1}^{4}+x_{2}^{4}+x_{3}^{4}$.

It follows that

$$
\begin{aligned}
\tau\left(x_{1}^{3} x_{2}^{3} y_{3} z\right) & =\tau\left(x_{1} x_{2} y_{3} z\right)\left(\lambda_{1} \tau\left(x_{1}^{4}\right)+\lambda_{2} \tau\left(x_{1}^{2} x_{2}^{2}\right)\right) \\
& +\tau\left(x_{1}^{2}\right)\left(\mu_{1} \tau\left(x_{1}^{3} x_{2} y_{3} z\right)+\mu_{2} \tau\left(x_{1} x_{2}^{3} y_{3} z\right)+\mu_{3} \tau\left(x_{1} x_{2} x_{3}^{2} y_{3} z\right)\right)
\end{aligned}
$$

for some $\lambda_{1}, \lambda_{2}, \mu_{1}, \mu_{2}, \mu_{3} \in \mathbb{F}$. Comparing the coefficients of $x_{1}^{5} x_{2} y_{3} z, x_{1} x_{2}^{5} y_{3} z$, $x_{1} x_{2} x_{3}^{4} y_{3} z, x_{1} x_{2}^{3} x_{3}^{2} y_{3} z$ we conclude

$$
0=\lambda_{1}+\mu_{1}=\lambda_{1}+\mu_{2}=\lambda_{1}+\mu_{3}=\lambda_{2}+\mu_{2}+\mu_{3} .
$$

It follows that the coefficient $\lambda_{2}+\mu_{1}+\mu_{2}$ of $x_{1}^{3} x_{2}^{3} y_{3} z$ on the right hand side is 0 , whereas on the left hand side it is 1 . This contradiction implies that $\tau\left(x_{1}^{3} x_{2}^{3} y_{3} z\right) \notin\left(R_{+}\right)^{2}$, hence $\beta\left(S(V)^{G}\right) \geq 8$.

Proposition 3.10. $\beta\left(\left(C_{2} \times C_{2}\right) \rtimes C_{4}\right)=6$.

Proof. We have $G=\left(C_{2} \times C_{2}\right) \rtimes\langle g\rangle$ where $\langle g\rangle \cong C_{4}$. The group $G$ contains the abelian normal subgroup $A:=C_{2} \times C_{2} \times\left\langle g^{2}\right\rangle$. We use the same notation for the elements of $\widehat{A}$ as in the proof of Proposition 3.8. The action of $\langle g\rangle$ on $\widehat{A}$ is given by

$$
(a, \varepsilon)^{g}=(b, \varepsilon), \quad(b, \varepsilon)^{g}=(a, \varepsilon), \quad(c, \varepsilon)^{g}=(c, \varepsilon), \quad(0, \varepsilon)^{g}=(0, \varepsilon) \quad \text { for } \varepsilon=0,1 .
$$

Take a zero-sum sequence $S$ over $\widehat{A}$ with $|S| \geq 7$ which is not the product of three non-empty zero-sum sequences. Since $\mathrm{D}(A)=4$ by Lemma 3.7 in [11, $S$ has no zero-sum subsequence of length at most 2 . It follows that $S$ consists of the non-zero elements of $\widehat{A}$ (each element having multiplicity 1 ), hence $S=S_{1} \cdot S_{2}$ where $S_{1}=\{(b, 0),(b, 1),(0,1)\}$ and $S_{2}=\{(a, 0),(a, 1),(c, 0),(c, 1)\}$. We have that

$$
S_{1} \cdot S_{2}^{g}=\{(b, 0),(b, 0)\} \cdot\{(b, 1),(b, 1)\} \cdot\{(c, 0),(c, 1),(0,1)\}
$$

is the product of $3=1+[G: A]$ zero-sum sequences. By Lemma 3.7 we conclude the desired inequality $\beta(G) \leq 6$.

On the other hand $\beta(G) \geq \beta\left(C_{2} \times C_{2}\right)+\beta\left(C_{4}\right)-1=3+4-1=6$ by ([6).

Example 3.11. For later use we present a $G$-module on which the Noether number of the group $G=\left(C_{2} \times C_{2}\right) \rtimes C_{4}$ is attained. Consider the $G$-module $V=\mathbb{F}^{4}$ on which the action is given by the matrices

$$
a=\left(\begin{array}{cccc}
1 & 0 & 0 & 0 \\
0 & -1 & 0 & 0 \\
0 & 0 & 1 & 0 \\
0 & 0 & 0 & -1
\end{array}\right), \quad b=\left(\begin{array}{cccc}
-1 & 0 & 0 & 0 \\
0 & 1 & 0 & 0 \\
0 & 0 & 1 & 0 \\
0 & 0 & 0 & -1
\end{array}\right), \quad c=\left(\begin{array}{cccc}
0 & 1 & 0 & 0 \\
1 & 0 & 0 & 0 \\
0 & 0 & \omega & 0 \\
0 & 0 & 0 & \omega
\end{array}\right)
$$


where $\omega$ is a primitive fourth root of unity. Then $a, b$ generate a subgroup of $G L(V)$ isomorphic to $C_{2} \times C_{2}, c^{4}$ is the identity matrix, and conjugation by $c$ interchanges $a$ and $b$. So $G$ can be identified with the subgroup of $G L\left(\mathbb{F}^{4}\right)$ generated by $a, b, c$. Moreover, the $2 \times 2$ upper left blocks of $a, b, c$ give the reflection representation of $\bar{G}=G /\left\langle c^{2}\right\rangle \cong D i h_{8}$ on $W=\mathbb{F}^{2} \subset V$. Denote by $x, y, z, w$ the standard basis vectors in $V=\mathbb{F}^{4}$, so $W=\operatorname{Span}_{\mathbb{F}}\{x, y\}, S(V)=$ $\mathbb{F}[x, y, z, w]$ and $S(W)=\mathbb{F}[x, y]$. We claim that the $G$-invariant

$$
x y\left(x^{2}-y^{2}\right) z w
$$

in $\mathbb{F}[x, y, z, w]^{G}$ is indecomposable. Consider the characters $\chi, \psi \in \widehat{G}$ given by

$$
\begin{aligned}
& \chi(a)=\chi(b)=\chi(c)=-1, \quad \psi_{1}(a)=\psi_{1}(b)=1, \psi_{1}(c)=\omega, \\
& \psi_{2}(a)=\psi_{2}(b)=-1, \psi_{2}(c)=\omega
\end{aligned}
$$

and $\mathbb{F}_{\chi}, \mathbb{F}_{\psi_{1}}, \mathbb{F}_{\psi_{2}}$ the corresponding one-dimensional $G$-modules. Clearly we have the $G$-module isomorphisms

$$
\mathbb{F} z \cong \mathbb{F}_{\psi_{1}}, \quad \mathbb{F} w \cong \mathbb{F}_{\psi_{2}}, \quad \mathbb{F} x y\left(x^{2}-y^{2}\right) \cong \mathbb{F}_{\chi}
$$

Since $c$ restricts to an order two transformation of $W$, the modules $\mathbb{F}_{\psi_{1}}, \mathbb{F}_{\psi_{2}}$ and their duals do not occur as a summand in $S(W)$, it follows that $S(V)^{G}$ contains no element that has degree 1 in $z$ and degree 0 in $w$, and $S(V)^{G}$ contains no element that has degree 1 in $w$ and degree 0 in $z$. The equalities $\psi_{1} \psi_{2}=\chi$ and $\chi^{2}=1$ show that the elements of $S(V)^{G}$ having degree 1 both in $z$ and $w$ are exactly the elements of the form $z w h$ where $h \in S(W)$ and $\mathbb{F} h \cong \mathbb{F}_{\chi}$. The $\bar{G}$-module structure of $S(W)$ and the structure of $S(V)^{G} \cap S(W)=S(W)^{\bar{G}}$ is well known from the Shephard-Todd-Chevalley Theorem [3], 33. We infer that $\mathbb{F}_{\chi}$ occurs with multiplicity one in the degree 4 component of $S(W)$, namely as the subspace spanned by $x y\left(x^{2}-y^{2}\right)$, and does not occur in lower degrees. This clearly implies that $x y\left(x^{2}-y^{2}\right) z w$ is indecomposable in $S(V)^{G}$.

\section{Observations and open questions}

\subsection{The Noether number and multiplicity free represen- tations}

When $\operatorname{char}(\mathbb{F})=0$, it was shown in 30 that as a consequence of Weyl's Theorem on polarizations, $\beta(G)$ is attained on the regular representation of $G$, which contains each irreducible $G$-module with multiplicity equal to its dimension (see 25] for a variant of Weyl's Theorem [35] valid in positive non-modular characteristic). However for the small groups studied here, the Noether number is usually attained on some $G$-modules of much smaller dimensions. Recall that a $G$-module $V$ is multiplicity free if it is the direct sum of pairwise non-isomorphic irreducible $G$-modules.

To deal with some particular cases below, we need first to state explicitly the following corollary of the proof of the inequality (6) given in [8]:

Lemma 4.1. Let $N$ be a normal subgroup of $G$ with $G / N$ abelian. For any $N$-module $W$ there exists a multiplicity free $G / N$-module $U$ such that

$$
\beta\left(S\left(U \oplus \operatorname{Ind}_{N}^{G} W\right)^{G}\right) \geq \beta\left(S(W)^{N}\right)+\mathrm{D}(G / N)-1 .
$$


In particular, if $\beta\left(S(W)^{N}\right)=\beta(N), \beta(G)=\beta(N)+\mathrm{D}(G / N)-1$, $\operatorname{Ind}_{N}^{G}(W)$ is multiplicity free and has no summand on which $N$ acts trivially, then $\beta(G)$ is attained on a multiplicity free $G$-module.

Theorem 4.2. There exists a multiplicity free $G$-module $V$ such that $\beta(G)=$ $\beta\left(S(V)^{G}\right)$ in each of the following cases:

1. $G$ is abelian;

2. $G$ has a cyclic subgroup of index two;

3. $G$ has order less than 32 .

Proof. It is sufficient to prove our claim in the case when $\mathbb{F}$ is algebraically closed, so let us assume this.

1. The case when $G$ is abelian is known. Denote by $\widehat{G}$ the group of characters ( $G \rightarrow \mathbb{F}^{\times}$homomorphisms) of $G$, and for $\chi \in \widehat{G}$ let $\mathbb{F}_{\chi}$ be the 1-dimensional $G$-module on which $G$ acts via $\chi$. It is well known (see for example Proposition 4.7 in [10]) that if there exists an irreducible zero-sum sequence over $\widehat{G}$ of length $\mathrm{D}(\widehat{G})$ with components in $\left\{\chi_{1}, \ldots, \chi_{k}\right\} \subseteq \widehat{G}$, then $\beta(G)=\beta\left(S\left(\bigoplus_{i=1}^{k} \mathbb{F}_{\chi_{i}}\right)^{G}\right)$.

2. For the dicyclic groups $G=D i c_{4 m}$ (where $m>1$ ) we gave an example in the proof of Proposition 9.1 in 8 of a 2-dimensional irreducible $G$-module $V$ with $\beta\left(S(V)^{D i c_{4 m}}\right)=2 m+2$.

Let $G$ be a non-abelian group with a cyclic subgroup $N$ of index two, and let $\chi$ be a generator of $\widehat{N}$. Then $\operatorname{Ind}_{N}^{G} \mathbb{F}_{\chi}$ is a 2-dimensional irreducible $G$-module, and so the module $U \oplus \operatorname{Ind}_{N}^{G} \mathbb{F}_{\chi}$ from Lemma 4.1 is multiplicity free (being the direct sum of a 2-dimensional irreducible and the non-trivial 1-dimensional module). Moreover, we have $\beta\left(S\left(U \oplus \operatorname{Ind}_{N}^{G} \mathbb{F}_{\chi}\right)^{G}\right) \geq \beta(N)+1=\frac{1}{2}|G|+1$, and $\frac{1}{2}|G|+1=\beta(G)$ unless $G$ is dicyclic.

3. Similar argument works for $C_{7} \rtimes C_{3}, C_{5} \rtimes C_{4}$, and $M_{27}$ : let $N$ be a maximal cyclic normal subgroup of the given group, and $\chi$ a generator of $\widehat{N}$. Then $\operatorname{Ind}_{N}^{G} \mathbb{F}_{\chi}$ is an irreducible $G$-module of dimension $|G / N|$, and we are done by Lemma 4.1, taking into account the known value of $\beta(G)$ from Section 2 ,

Now suppose that $G=A \rtimes_{-1} C_{2}$ is a generalized dihedral group with $A$ being a non-trivial abelian group. Take a minimal subset $\Lambda=\left\{\chi_{1}, \ldots, \chi_{r}\right\} \subset \widehat{A}$ such that $\mathcal{B}(\widehat{A})$ contains an atom of length $\mathrm{D}(\widehat{A})$, all of whose components belong to $\Lambda$. By minimality of $\Lambda$, it does not contain the trivial character, and if $\chi$ and $\chi^{-1}$ both belong to $\Lambda$, then $\chi=\chi^{-1}$. If $\chi \neq \chi^{-1}$, then $\operatorname{Ind}_{A}^{G} \mathbb{F}_{\chi}$ is irreducible and as an $A$-module is isomorphic to $\mathbb{F}_{\chi} \oplus \mathbb{F}_{\chi^{-1}}$, whereas if $\chi=\chi^{-1}$ is non-trivial, then $\operatorname{Ind}_{A}^{G} \mathbb{F}_{\chi}$ is the direct sum of two non-isomorphic 1-dimensional $G$-modules, which as $A$-modules are isomorphic to $\mathbb{F}_{\chi}$. It follows that for $W=\mathbb{F}_{\chi_{1}} \oplus \cdots \oplus \mathbb{F}_{\chi_{r}}$ the $G$-module $\operatorname{Ind}_{A}^{G} W$ is multiplicity free and contains no summands on which $A$ acts trivially. Thus we are done by Lemma 4.1

The groups $Q_{8} \times C_{2}, S_{3} \times C_{3}, C_{4} \rtimes C_{4}$, can also be settled by Lemma 4.1 . taking into account the known value of $\beta(G)$ from Section 22. Indeed, for the 2-dimensional irreducible $Q_{8}$-module $W$ we have $\beta(S(W))^{Q_{8}}=6$ (see 30 ) and $\operatorname{Ind}_{Q_{8}}^{Q_{8} \times C_{2}}$ is the direct sum of the two non-isomorphic irreducible twodimensional $Q_{8} \times C_{2}$-modules. Essentially the same argument can be used for $D i c_{12} \times C_{2}$ : after inducing up to $D i c_{12} \times C_{2}$ the irreducible two-dimensional Dic $c_{12}$-module on which the Noether number is attained we get a direct sum of two non-isomorphic irreducible $\operatorname{Dic}_{12} \times C_{2}$-modules. Note that $S_{3} \times C_{3} \cong$ 
$C_{3} \rtimes_{-1} C_{6}$ and for a non-trivial $\chi \in \widehat{C}_{3}$ we have that $\operatorname{Ind}_{C_{3}}^{C_{3} \rtimes C_{6}} \mathbb{F}_{\chi}$ is the direct sum of the three pairwise non-isomorphic irreducible 2-dimensional $S_{3} \times C_{3}$ modules. For a generator $\chi \in \widehat{C}_{4}$, we have that $\operatorname{Ind}_{C_{4}}^{C_{4} \rtimes C_{4}} \mathbb{F}_{\chi}$ is the direct sum of two non-isomorphic irreducible two-dimensional modules.

The cases of the groups $C_{3} \rtimes D i h_{8}, A_{4} \times C_{2}$ and $\left(C_{2} \times C_{2}\right) \rtimes_{-1} C_{4}$ were settled in Example 3.6. Example 3.9 and Example 3.11.

An irreducible module on which the Noether number is attained is given already in the literature for $A_{4}, \widetilde{A}_{4}$ in [7, for $H_{27}$ in [5]. It was pointed out in [10. Example 5.3 and 5.4] that the Noether number for $S_{4}$ is attained on the product of the standard four-dimensional permutation representation and the sign representation, and for the Pauli group $\left(C_{4} \times C_{2}\right) \rtimes_{\phi} C_{2}$ on the direct sum of the two-dimensional pseudo-reflection representation and a one-dimensional representation (see also [6] for some details referred to in [10]).

Problem 4.3. Does there exist a group $G$ for which $\beta\left(S(V)^{G}\right)<\beta(G)$ for all multiplicity free $G$-modules $V$ ?

Remark 4.4. (i) By a theorem of Draisma, Kemper and Wehlau 12 the universal degree bound for separating invariants is known to be attained on multiplicity free representations.

(ii) We mention a conjecture of Hunziker [23, Conjecture 5.1] made for reflection groups that has a similar flavor as the topic of Section 4.1.

\subsection{The strict monotonicity of the Noether number}

Since all $G / N$-modules can be viewed as $G$-modules, the inequality $\beta(G / N) \leq$ $\beta(G)$ holds for any normal subgroup $N$ of any finite group $G$. It was proven by B. Schmid [30] that $\beta(H) \leq \beta(G)$ for any subgroup $H$ of $G$. We shall refer as $\mathbf{S}$ and $\mathbf{F}$ for the following conditions on a finite group $G$ :

S: $\beta(H)<\beta(G)$ for each proper subgroup $H$ of $G$.

F: $\beta(G / N)<\beta(G)$ for each non-trivial normal subgroup $N$ of $G$.

It is shown in a subsequent paper 9 that conditions $\mathbf{S}$ and $\mathbf{F}$ (by generalizing (6) for non-abelian $N$ ) hold for all finite groups. We collect in Theorem 4.5 below facts on the properties $\mathbf{S}$ and $\mathbf{F}$ that can be read off from the results obtained or quoted in the present paper.

Theorem 4.5. Condition $\mathbf{S}$ holds for any finite nilpotent group G. Moreover, both $\mathbf{S}$ and $\mathbf{F}$ hold when

1. $G$ is abelian;

2. G has a cyclic subgroup of index two;

3. $G \cong C_{p} \rtimes C_{q}$ for odd primes $p, q$ where $q \mid p-1$;

4. $G$ has order less than 32 .

Proof. Suppose first that $G$ is nilpotent, and let $H$ be a proper subgroup of $G$. It is well known that $H$ is contained as a prime index normal subgroup in a subgroup $K$ of $G$, whence we have $\beta(G) \geq \beta(K) \geq \beta(H)+[K: H]-1>\beta(H)$ by (6). So $\mathbf{S}$ holds for $G$. Note that if $G$ is abelian, then any factor group 
of $G$ is isomorphic to a subgroup of $G$, hence $\mathbf{F}$ follows from $\mathbf{S}$. If $G$ has a cyclic subgroup of index two, then any subgroup or factor group of $G$ has a subgroup of index at most two as well, whence (3) shows that both $\mathbf{S}$ and $\mathbf{F}$ hold. Any non-trivial subgroup or factor group of $C_{p} \rtimes C_{q}$ has order $p$ or $q$, and $\beta\left(C_{p} \rtimes C_{q}\right) \geq p+q-1$ by (6).

Assume finally that $G$ is a non-abelian group of order less than 32 that contains no cyclic subgroup of index two. Theorem 1.1 in [7] asserts that $\beta(H)<$ $\frac{1}{2}|H|$ unless $H$ has a cyclic subgroup of index at most two, or $H$ is isomorphic to one of $C_{2} \times C_{2} \times C_{2}, C_{3} \times C_{3}, A_{4}$, or $\tilde{A}_{4}$. Taking into account (3) and the values of the Noether numbers of $C_{2} \times C_{2} \times C_{2}, C_{3} \times C_{3}, A_{4}$ and $\tilde{A}_{4}$, this implies that $\beta(H) \leq 2+\frac{1}{2}|H|$ for any non-cyclic $H$, with equality only if $H \cong D i c_{4 m}$ is a dicyclic group. Since our $G$ has no cyclic subgroups or factor groups of order at least $\frac{1}{2}|G|$, we conclude that for any proper subgroup or factor group $H$ of $G$ the inequality $\beta(H) \leq \max \left\{2+\frac{1}{4}|G|, \frac{1}{3}|G|\right\}$ holds, with strict inequality unless $H \cong D i c_{4 m}$ is a dicyclic group of order $\frac{1}{2}|G|$ or $H$ is a cyclic group of order $\frac{1}{3}|G|$. This immediately implies that $\mathbf{S}$ and $\mathbf{F}$ hold for $G$ provided that $\beta(G)>\max \left\{2+\frac{1}{4}|G|, \frac{1}{3}|G|\right\}$. From now on assume in addition that $\beta(G) \leq \max \left\{2+\frac{1}{4}|G|, \frac{1}{3}|G|\right\}$. So $G$ is one of the following groups from the table in Section 2 the two groups of order 16 with Noether number 6 , the group of order 18 with Noether number 6, the two groups of order 24 with Noether number 8 , or the group of order 27 with Noether number 9 . Now $D i h_{8} \times C_{2}$ has exactly four elements of order 4 and no element of order 8 , consequently does not have $Q_{8}=D i c_{8}$ as a subgroup or a factor group, hence $\mathbf{S}$ and $\mathbf{F}$ hold for this group. The group $\left(C_{2} \times C_{2}\right) \rtimes C_{4}$ has $C_{2} \times C_{2} \times C_{2}$ as a subgroup. Therefore any order 8 subgroup or factor group of this group contains $C_{2} \times C_{2}$ as a subgroup, and hence it is not dicyclic. The group $\left(C_{3} \times C_{3}\right) \rtimes_{-1} C_{2}$ has no element of order 6 and has no dicyclic subgroups or factor groups (as its order is not divisible by 4 ). The groups $A_{4} \times C_{2}$ and $D i h_{12} \times C_{2}$ have no element of order 8 and do not have a subgroup or factor group isomorphic to Dic ${ }_{12}$ (as these groups do not have an element of order 4). Finally, the Heisenberg group does not have an element of order 9 . So $\mathbf{S}$ and $\mathbf{F}$ hold for all groups of order less than 32 .

\subsection{Dependence on the characteristic}

It is proved in [25, Corollary 4.2$]$ that $\beta^{\mathbb{F}}(G)$ may depend only on the characteristic of $\mathbb{F}$, but not on $\mathbb{F}$. Therefore we introduce the notation

$$
\beta^{\operatorname{char}(\mathbb{F})}(G)=\beta^{\mathbb{F}}(G) .
$$

Moreover, by [25, Theorem 4.7] we have $\beta^{p}(G) \geq \beta^{0}(G)$ for all primes $p$, and $\beta^{p}(G)=\beta^{0}(G)$ holds for all but finitely many primes $p$. Knop remarks in 25 that "Presently, no group $G$ and prime $p$ not dividing $|G|$ with $\beta^{p}(G)>\beta^{0}(G)$ seems to be known". This observation inspires the following question:

Problem 4.6. Does the equality $\beta^{p}(G)=\beta^{0}(G)$ hold for all finite groups $G$ and primes $p$ not dividing $|G|$ ?

The paper 34 reports as a folklore conjecture that for any permutation $\mathbb{Z} G$ module $V$ (i.e. when $V$ is a free $\mathbb{Z}$-module with a basis preserved by the action of $G)$ we have $\beta\left(S\left(\mathbb{F} \otimes_{\mathbb{Z}} V\right)^{G}\right)=\beta\left(S\left(\mathbb{Q} \otimes_{\mathbb{Z}} V\right)^{G}\right)$ provided that $\operatorname{char}(\mathbb{F})$ does 
not divide $|G|$. We note that if this conjecture is true, then Problem 4.6 has a positive answer. Indeed, it follows from [25, Theorem 6.1] and (1) that if $p$ does not divide $|G|$ then $\beta^{p}(G)=\beta\left(S\left(\mathbb{F} \otimes_{\mathbb{Z}} V\right)^{G}\right)$ where $\mathbb{F}$ is a field of characteristic $p$ and $V$ is the direct sum of $|G|$ copies of the regular $G$-module defined over $\mathbb{Z}$ (hence in particular $V$ is a permutation $\mathbb{Z} G$-module).

In Theorem 4.7 below we collect the cases for which Problem 4.6 has a positive answer by the results obtained or quoted in the present paper.

Theorem 4.7. The equality $\beta^{p}(G)=\beta(G)$ holds for all primes $p$ not dividing the order of $G$ in each of the following cases:

1. $G$ is abelian;

2. G has a cyclic subgroup of index two;

3. G has order less than 32 .

Proof. It has been long known that if $G$ is abelian then $\beta^{p}(G)=\mathrm{D}(G)$ for all $p \nmid|G|$. Formula (3) for the Noether number of a group with a cyclic subgroup of index two is valid in all non-modular characteristic. Finally, the quantities in the table in Section 2 are independent of the characteristic of the base field (provided that it is non-modular), whence the statement holds also for the remaining groups of order less than 32 .

\subsection{Further observations}

Remark that the groups $S_{3} \times C_{3}$ and $\left(C_{3} \times C_{3}\right) \rtimes_{-1} C_{2}$ both have the structure $\left(C_{3} \times C_{3}\right) \rtimes_{\alpha} C_{2}$, the only difference being in the automorphism $\alpha$. However, their Noether numbers are different.

The groups $D i h_{8} \times C_{2},\left(C_{2} \times C_{2}\right) \rtimes C_{4}$ and the Pauli group all have the structural description $\left(C_{4} \times C_{2}\right) \rtimes_{\alpha} C_{2}$, only the automorphism $\alpha$ being different in the three cases. The Noether numbers of the first two are equal, and differ from the Noether number of the third one.

This shows that it would be interesting to understand how $\beta\left(A \rtimes_{\alpha} B\right)$ depends on $\alpha$.

\section{Davenport constants}

\subsection{The monoid of product-one sequences}

In this section we introduce further notation related to the small and large Davenport constants of a not necessarily abelian finite group. We follow the presentation of [10].

Let $G_{0} \subseteq G$ be a non-empty subset of a finite group $G$. A sequence over $G_{0}$ means a finite sequence of terms from $G_{0}$ which is unordered, and repetition of terms is allowed (in other words, a sequence over $G_{0}$ is a multiset of elements from $G_{0}$ ). A sequence will be considered as an element of the free abelian monoid $\mathcal{F}\left(G_{0}\right)$ whose generators are identified with the elements of $G_{0}$. We use the symbol "." for the multiplication in the monoid $\mathcal{F}\left(G_{0}\right)$ - this agrees with the convention in the monographs [19, 21 - and we denote multiplication in $G$ by juxtaposition of elements. For example, considering elements $g_{1}, g_{2} \in G_{0}$ we 
have that $g_{1} \cdot g_{2} \in \mathcal{F}\left(G_{0}\right)$ is a sequence of length 2 , while $g_{1} g_{2}$ is an element of $G$. Furthermore, we use brackets for the exponentiation in $\mathcal{F}\left(G_{0}\right)$. So for $g \in G_{0}, S \in \mathcal{F}\left(G_{0}\right)$, and $k \in \mathbb{N}_{0}$, we have

$$
g^{[k]}=\underbrace{g \cdot \ldots \cdot g}_{k} \in \mathcal{F}(G) \quad \text { and } \quad S^{[k]}=\underbrace{S \cdot \ldots \cdot S}_{k} \in \mathcal{F}(G) .
$$

Let

$$
S=g_{1} \cdot \ldots \cdot g_{|S|}=\prod_{g \in G_{0}} g^{\left[\mathrm{v}_{g}(S)\right]}
$$

be a sequence over $G_{0}$; here $\vee_{g}(S)$ is the multiplicity of $g$ in $S$ and we call $|S|=\sum_{g \in G} \vee_{g}(S)$ the length of $S$. If $\vee_{g}(S)>0$, i.e. $S=g \cdot R$ for a sequence $R$ with $|R|=|S|-1$, then we write

$$
S \cdot g^{[-1]}=R
$$

for the sequence obtained from $S$ by removing one occurrence of $g$. More generally, we write $R=S \cdot T^{[-1]}$ if we have $S=R \cdot T$ for some sequences $R, S, T$. The identity element $1_{\mathcal{F}\left(G_{0}\right)}$ in $\mathcal{F}\left(G_{0}\right)$ is called the trivial sequence, and has length $\left|1_{\mathcal{F}\left(G_{0}\right)}\right|=0$. We have the usual divisibility relation in the free abelian monoid $\mathcal{F}\left(G_{0}\right)$ and write $T \mid S$ if $T$ divides $S$. A divisor $T$ of $S$ will also be called a subsequence of $S$. We call $\operatorname{supp}(S)=\left\{g \in G_{0} \mid \mathrm{v}_{g}(S)>0\right\} \subseteq G_{0}$ the support of $S$. The set of products of $S$ is

$$
\pi(S)=\left\{g_{\tau(1)} \ldots g_{\tau(|S|)} \in G \mid \tau \in \operatorname{Sym}\{1, \ldots,|S|\}\right\} \subseteq G
$$

(if $|S|=0$, we use the convention that $\pi(S)=\left\{1_{G}\right\}$ ). Clearly, $\pi(S)$ is contained in a $G^{\prime}$-coset, where $G^{\prime}=[G, G]=\left\langle g^{-1} h^{-1} g h \mid g, h \in G\right\rangle$ denotes the commutator subgroup of $G$. Set

$$
\Pi(S)=\bigcup_{\substack{T \mid S \\ 1_{\mathcal{F}}\left(G_{0}\right) \neq T}} \pi(T) \subseteq G .
$$

The sequence $S$ is called a

- product-one sequence if $1_{G} \in \pi(S)$,

- product-one free sequence if $1_{G} \notin \Pi(S)$.

The set

$$
\mathcal{B}\left(G_{0}\right)=\left\{S \in \mathcal{F}\left(G_{0}\right): 1_{G} \in \pi(S)\right\}
$$

of all product-one sequences over $G_{0}$ is obviously a submonoid of $\mathcal{F}\left(G_{0}\right)$. We denote by $\mathcal{A}\left(G_{0}\right)$ the set of atoms in the monoid $\mathcal{B}\left(G_{0}\right)$. The length of an atom is clearly bounded by $|G|$. The large Davenport constant of $G_{0}$ is

$$
\mathrm{D}\left(G_{0}\right)=\max \left\{|S|: S \in \mathcal{A}\left(G_{0}\right)\right\} \in \mathbb{N} .
$$

Moreover, we denote by $\mathcal{M}\left(G_{0}\right)$ the set of product-one free sequences over $G_{0}$ and we define the small Davenport constant of $G_{0}$ as

$$
\mathrm{d}\left(G_{0}\right)=\max \left\{|S|: S \in \mathcal{M}\left(G_{0}\right)\right\} .
$$

We have the inequality

$$
\mathrm{d}\left(G_{0}\right)+1 \leq \mathrm{D}\left(G_{0}\right)
$$

with equality when the elements in $G_{0}$ commute with each other. 


\subsection{Some known results}

For a non-cyclic group $G$ with a cyclic subgroup of index two Olson and White [28. proved that $\mathrm{d}(G)=\frac{1}{2}|G|$. Morover, recently it was proven by Geroldinger and Grynkiewicz [18] that for these groups $\mathrm{D}(G)=\mathrm{d}(G)+\left|G^{\prime}\right|$.

For the non-abelian semidirect product $C_{p} \rtimes C_{q}$ where $p, q$ are odd primes it was shown by Grynkiewicz [20, Corollary 5.7 and Theorem 5.1] that we have $\mathrm{d}\left(C_{p} \rtimes C_{q}\right)=p+q-2$ and $\mathrm{D}\left(C_{p} \rtimes C_{q}\right)=2 p$.

\subsection{The large Davenport constant for $H_{27}$}

Consider $H_{27}$, the Heisenberg group with 27 elements having the presentation $\left\langle a, b, c \mid a^{3}=b^{3}=c^{3}=1, c=[a, b]=a^{-1} b^{-1} a b\right\rangle$. This is an extraspecial group, its commutator subgroup $\langle c\rangle$ coincides with the center $Z:=Z\left(H_{27}\right)$. As a result, the commutator identities (which hold for any group) take the following simpler form in this particular case:

$$
[x, y z]=[x, y][x, z] \quad[x y, z]=[x, z][y, z]
$$

for any $x, y, z \in H_{27}$. As $[c, x]=1$ for any $x \in H_{27}$ we see that the value of $[x, y]$ depends only on the $\operatorname{cosets} x Z$ and $y Z$ so that the commutator defines in fact a bilinear map on $H_{27} / Z \cong C_{3} \times C_{3}$ with values in $C_{3}$. Moreover as $c$ commutes with every other element of the group it is immediate that every $Z$ coset has a representative of the form $a^{i} b^{j}$ for some $i, j \in \mathbb{Z} / 3 \mathbb{Z}$ and by repeated applications of (9) we get

$$
\left[a^{i} b^{j}, a^{k} b^{l}\right]=c^{i l-j k}=c^{\operatorname{det}\left(\begin{array}{ll}
i & j \\
k & l
\end{array}\right)} .
$$

By (10) the elements $x=a^{i} b^{j}$ and $y=a^{k} b^{l}$ commute if and only if the vectors $(i, j)$ and $(k, l)$ are linearly dependent over $\mathbb{Z} / 3 \mathbb{Z}$. For the rest we denote by $\bar{x}$ the image of any $x \in H_{27}$ at the natural surjection $H_{27} \rightarrow C_{3} \times C_{3}$ and we extend this notation to sequences in the obvious way, as well.

We say that two sequences $S$ and $T$ over a group $G$ are similar if $\alpha(S)=T$ for an automorphism $\alpha \in \operatorname{Aut}(G)$ (the action of $\operatorname{Aut}(G)$ on $G$ extends naturally to an action on $\mathcal{F}(G)$ ). A sequence $S$ over $G$ is called degenerate if $\operatorname{supp}(S)$ is contained in a proper subgroup of $G$.

Lemma 5.1. Let $T=R \cdot S$ be a product-one sequence such that $|\pi(R)|=3$ and $S$ is not product-one free. Then $T$ is not an atom.

Proof. By the assumption on $S$ there is a non-empty product-one sequence $U \mid S$. Consider the sequence $V=T \cdot U^{[-1]}$. Then $\bar{V}$ is a zero-sum sequence over $C_{3} \times C_{3}$, whence $\pi(V) \subseteq Z$. But for $R \mid V$ we have $|\pi(R)|=3$ hence $\pi(V)=Z$ so that $V$ is also a product-one sequence. Thus the equality $T=U \cdot V$ shows that $T$ is not an atom.

Lemma 5.2. Let $T$ be a non-degenerate sequence over $H_{27} \backslash Z$ of length at least 3. Then either $|\pi(T)|=3$ or $\bar{T}=\bar{e} \cdot \bar{f} \cdot(-\bar{e}-\bar{f})$ for a basis $\{\bar{e}, \bar{f}\}$ of $H_{27} / Z$

Proof. Assume that $\pi(T)$ is not a full $Z$-coset. As $T$ is non-degenerate there must be two elements $e, f$ in $T$ such that $[e, f]=c$. Hence $|\pi(T)|=2$. Let $g$ be an arbitrary element in $T \cdot(e \cdot f)^{[-1]}$. Then $\bar{g} \neq \bar{e}$ because otherwise 
$\pi(g \cdot e \cdot f) \supseteq\{g e f, g f e, f g e\}=g e f Z$. Also $\bar{g} \neq-\bar{e}$ because otherwise $\pi(g \cdot e \cdot f) \supseteq$ $\{g e f, g f e, e f g\}=$ gef $Z$. Similarly $\bar{g}$ is different from $\bar{f},-\bar{f}$.

As a result in the $\mathbb{Z} / 3 \mathbb{Z}$-vector space $H_{27} / Z \cong C_{3} \times C_{3}$ we have a relation $\alpha \bar{e}+\beta \bar{f}+\gamma \bar{g}=0$ where the coefficients $\alpha, \beta$ are non-zero. Moreover $\gamma \neq 0$ also holds by the linear independence of $\bar{e}$ and $\bar{f}$. Up to similarity and the choice of the basis $e, f$ only two cases are possible: (i) $\bar{e}+\bar{f}=\bar{g}$, but then $[e, f]=[e, g]=c$, hence $\pi(e \cdot f \cdot g)=e f g Z$, again a contradiction, or (ii) $\bar{e}+\bar{f}+\bar{g}=0$; then $[e, f]=[g, e]=[f, g]=c$ hence $e f g=c f e g=f g e=c g f e=g e f=c e g f$, so that $|\pi(e \cdot f \cdot g)|=2$.

In the proof below we shall use the following ad hoc terminology: A subset of $C_{3} \times C_{3}$ of the form $\{e, f,-e-f\}$ where $e, f$ form a basis of $C_{3} \times C_{3}$ will be called an affine line, while a subset of the form $\{e, f, e+f\}$ where $e, f$ form a basis of $C_{3} \times C_{3}$ will be called an affine cap (the terminology is motivated by the literature on the so-called cap set problem). Note that a three-element subset of $C_{3} \times C_{3}$ in which any two elements are linearly independent over $\mathbb{Z} / 3 \mathbb{Z}$ is either an affine line or an affine cap.

Proposition 5.3. $\mathrm{D}\left(H_{27}\right) \leq 8$.

Proof. Assume indirectly that there is an atomic product-one sequence $T$ of length at least 9 . After ordering the elements of $T=g_{1} \cdot g_{2} \cdots g_{n}$ in such a way that $g_{1} g_{2} \cdots g_{n}=1$ and replacing $T$ with $T^{\prime}=g_{1} \cdots g_{8} \cdot\left(g_{9} g_{10} \cdots g_{n}\right)$ we may assume that $|T|=9$.

A. If $T$ is degenerate then it is in fact an irreducible zero-sum sequence over $C_{3} \times C_{3}$, hence $|T| \leq \mathrm{D}\left(C_{3} \times C_{3}\right)=5$, a contradiction. So for the rest we assume that $T$ is non-degenerate so that $\bar{T}$ contains a basis $\{e, f\}$ of $H_{27} / Z$.

B. $\bar{T}$ contains an element $g \notin\langle e\rangle \cup\langle f\rangle$ (i.e. $\bar{T}$ has an affine line or an affine cap as a subsequence). Otherwise, if $\bar{T}$ is contained in the set $\langle e\rangle \cup\langle f\rangle$ then $T=C \cdot A_{1} \cdot \ldots \cdot A_{t}$ where $\bar{C}=0^{[k]}$ and $\bar{A}_{i} \in\left\{e^{[3]}, f^{[3]},-e \cdot e,-f \cdot f\right\}$. Choose $a_{i} \in \pi\left(A_{i}\right)$ for $i=1, \ldots, t$. Then the sequence $Q:=C \cdot a_{1} \cdot \ldots \cdot a_{t}$ is a zero-sum sequence over $Z \cong C_{3}$, hence if $k+t>3$ then $Q$ factors into the product of two non-empty zero-sum sequences and $T$ factors accordingly, a contradiction. If $k>0$ then we get $k+t \geq k+(9-k) / 3>3$, as $\left|A_{i}\right| \leq 3$ for all $i$, again a contradiction. Hence $k=0, t=3$ and $\bar{T}$ is similar to $e^{[6]} \cdot f^{[3]}$. Then $T$ contains a degenerate subsequence of length $6>\mathrm{D}\left(C_{3} \times C_{3}\right)$, which in turn must contain a proper zero-sum subsequence $R$ such that $\bar{R}=e^{[3]}$. Then the complement $S=T \cdot R^{[-1]}$ has $|S|=6$, hence $\pi(S)=Z$ by Lemma [5.2, a contradiction by Lemma [5.1.

C. $\bar{T}$ cannot contain a subsequence $\bar{R} \mid \bar{T}$ similar to one of the following sequences:

$$
\begin{aligned}
& e \cdot f \cdot(-e) \cdot(-f) \\
& e \cdot f \cdot(e+f)^{[2]}
\end{aligned}
$$

Indeed, these sequences $\bar{R}$ are such that for their preimages $R$ we have that $\pi(R)=Z$ by Lemma 5.2, So for any such $R \mid T$ the complement $S=T$. $R^{[-1]}$ must be product-one free, as otherwise we would get a contradiction by Lemma 5.1 Hence $S$ cannot be degenerate, as $|S|=5=\mathrm{D}\left(C_{3} \times C_{3}\right)$ and $\pi(S) \subseteq Z \backslash\{1\}$. Therefore by Lemma 5.2 it is necessary that $S=x \cdot y \cdot L$ where 
$\bar{x}=\bar{y}=0$ and $\bar{L}$ is an affine line. Moreover by our assumption we must have $\pi(L)=Z \backslash\{1\}$ and $x, y \in Z \backslash\{1\}$, as well. But then $1 \in \pi(x \cdot L)$, a contradiction.

D. We claim that $\bar{T}$ must contain an affine line and $0 \notin \operatorname{supp}(\bar{T})$. For assume that $\bar{T}$ does not contain an affine line. Then $\bar{T}$ still contains an affine cap by $\mathbf{B}$, so we may assume that $e \cdot f \cdot(e+f) \mid T$. Then to avoid affine lines it is necessary that $e-f, f-e,-e-f$ do not occur in $\bar{T}$. To avoid subsequences of type (111) we have either $v_{\bar{T}}(-e)=0$ or $v_{\bar{T}}(-f)=0$. Assume now that $\mathrm{v}_{\bar{T}}(-e)>0$ and $\mathrm{v}_{\bar{T}}(-f)=0$ (the case $\mathrm{v}_{\bar{T}}(-e)=0$ and $\mathrm{v}_{\bar{T}}(-f)>0$ is analogous). Then to avoid subsequences of type (12) we must have $\mathrm{v}_{\bar{T}}(f)=\mathrm{v}_{\bar{T}}(e+f)=1$. As a result the image of $\bar{T}$ modulo $\langle e\rangle$ is $f^{[2]} \cdot 0^{[7]}$, but this is not a product-one sequence in $C_{3} \times C_{3} /\langle e\rangle \cong C_{3}$, a contradiction.

So it remains that $\bar{T}=0^{[k]} \cdot e^{[i]} \cdot f^{[j]} \cdot(e+f)$. Since $\bar{T}$ is a zero-sum sequence over $C_{3} \times C_{3}$ we must have $i \equiv j \equiv 2(\bmod 3)$. As $|\bar{T}|=9$ either $k+i=6$ or $k+j=6$ and we get again a degenerate subsequence which must contain a zerosum subsequence $R$ such that its complement $S=T \cdot R^{[-1]}$ has $\pi(S)=Z$ by Lemma 5.2, again a contradiction. Thus $\bar{T}$ contains an affine line. An argument similar to the one in $\mathbf{C}$ shows also that 0 does not occur in $\bar{T}$.

From now on we assume that $\bar{T}$ contains the affine line $\bar{L}=\{e, f, g\}$ as a subsequence.

E. Next we claim that $\sum_{x \in-\bar{L}} \mathbf{v}_{\bar{T}}(x) \leq 1$. Indeed, $\bar{T}$ cannot contain two different elements $x, y$ belonging to $-\bar{L}$ because that would yield a subsequence of type (11). Moreover, $\mathrm{v}_{\bar{T}}(x) \geq 2$ for some $x$ in $-L$ would yield the type (12) subsequence $x \cdot x \cdot L \cdot(-x)^{[-1]}$ of $\bar{T}$.

F. We claim that $\mathbf{v}_{\bar{T}}(e-f)=0$ and $\mathbf{v}_{\bar{T}}(f-e)=0$. For assume to the contrary that $\mathbf{v}_{\bar{T}}(e-f)>0$ (the case $\mathbf{v}_{\bar{T}}(f-e)>0$ being analogous). Observe that for any $x$ in $L$ we must have $\mathrm{v}_{\bar{T}}(x)=1$ to avoid subsequences of type (12). In view of $\mathbf{E}$ it follows that $\mathbf{v}_{\bar{T}}(e-f)+\mathrm{v}_{\bar{T}}(f-e) \geq|T|-4=5=\mathrm{D}\left(C_{3} \times C_{3}\right)$, so that $T$ has again a degenerate subsequence containing a zero-sum subsequence $R($ with $\operatorname{supp}(\bar{R}) \subseteq\{0, e-f, f-e\})$ such that its complement $S=T \cdot R^{[-1]}$ has $\pi(S)=Z$ by Lemma 5.2, leading to a contradiction by Lemma 5.1

G. Now we prove that $\operatorname{supp}(\bar{T})=L$. For otherwise it would follow from D, E, $\mathbf{F}$ that $\bar{T}=(-x) \cdot x \cdot \bar{T}_{0}$ where $\mathrm{v}_{L}(x)>0$ and $\operatorname{supp}\left(\bar{T}_{0}\right) \subseteq L$. But $\bar{T}_{0}$ must also be a zero-sum sequence, hence $\left|\bar{T}_{0}\right|$ is divisible by 3 because the only irreducible zero-sum sequences over $C_{3} \times C_{3}$ with support contained in $L$ are $e^{[3]}, f^{[3]},(-e-f)^{[3]}, e \cdot f \cdot(-e-f)$. But then $|T| \equiv 2 \bmod 3$, contradicting the assumption that $|T|=9$.

H. So we have a factorization $T=L \cdot R$ such that $\bar{L}=e \cdot f \cdot g$ with $e+f+g=0 \in C_{3} \times C_{3}$ and $\operatorname{supp}(\bar{R}) \subseteq\{e, f, g\}$. For any such factorization $\bar{L}$ and $\bar{R}$ are zero-sum sequences, hence $\pi(R) \subseteq Z$, moreover $|\pi(R)|=3$ by Lemma [5.2, so that $1 \in \pi(R)$ and consequently $1 \notin \pi(L)$. Observe now that an element in $\operatorname{supp}(\bar{T})$, say $e$ must have $\mathbf{v}_{\bar{T}}(e) \geq 3$. Let $Q=x_{1} \cdot x_{2} \cdot x_{3} \mid T$ be such that $\bar{Q}=e^{[3]}$ and $x_{1} \in L$. If $x_{i} \neq x_{1}$ for some $i>1$ then consider the sequence $L^{\prime}:=L \cdot x_{1}^{[-1]} \cdot x_{i}$. By assumption $\pi(L)=Z \backslash\{1\}$ and $x_{1}^{-1} x_{i} \in Z \backslash\{1\}$ hence $\pi\left(L^{\prime}\right)=\pi(L) x_{1}^{-1} x_{i} \ni 1$. Thus $\bar{L}^{\prime}=\bar{L}$ and $T=L^{\prime} \cdot R^{\prime}$ would be a factorization of $T$ as a product of two product-one sequences. We conclude that $x_{1}=x_{2}=x_{3}$, so that $Q$ is a product-one subsequence and $T=Q \cdot S$ where $\bar{S}$ is a product-one sequence over $C_{3} \times C_{3}$ and hence $S$ is a product-one sequence by Lemma 5.2. This contradicts the assumption that $T$ is an atom.

Remark 5.4. The idea of Lemma 5.1 appears in the proof of a result of 
Grynkiewicz 20, Corollary 3.4] which yields for the Heisenberg group of order $p^{3}$ the inequality

$$
\mathrm{D}(G) \leq \mathrm{d}(G)+\left|G^{\prime}\right|=\mathrm{d}(G)+p .
$$

We established by our algorithm that $\mathrm{d}\left(H_{27}\right)=6$ so in this case (13) gives $\mathrm{D}(G) \leq 9$ which in view of Proposition 5.3 is not sharp. On the other hand we show below that Proposition 5.3 is sharp.

Proposition 5.5. $\mathrm{D}\left(H_{27}\right) \geq 8$.

Proof. Assume $c \in \pi\left(a^{[i]} \cdot b^{[j]}\right)$ for some $i, j \in \mathbb{N}$. Then $i, j>0$ since $c \notin\langle a\rangle$ and $c \notin\langle b\rangle$. Moreover $\bar{c}=0=\bar{a}^{i} \bar{b}^{j}$ holds, as well. But since $\bar{a}$ and $\bar{b}$ are independent in $C_{3} \times C_{3}$ it is necessary that $i \equiv j \equiv 0 \bmod 3$. It follows that $i+j \geq 6$. On the other hand $c=[a, b]=a a b b a b$ hence the minimal expression of $c$ in terms of $a$ and $b$ has length 6 . By a similar argument the same is true for $c^{-1}$, as well.

Now $c c b b a a b a=1$ and we claim that the product-one sequence $S=a^{[3]}$. $b^{[3]} \cdot c^{[2]}$ is an atom. Otherwise let $S=T \cdot R$ for some non-empty product-one sequences $T, R$ and we may assume that $\mathrm{v}_{T}(c)>0$. If $\mathrm{v}_{R}(c)>0$ holds, too, then $T \cdot c^{[-1]}$ contains only $a, b$ and $c^{-1} \in \pi\left(T \cdot c^{[-1]}\right)$. Hence, by what has been said before, $T \cdot c^{[-1]}=a^{[3]} \cdot b^{[3]}$. But then $R=c$, which is not a product-one sequence. From this contradiction it follows that $\mathrm{v}_{R}(c)=0$ hence $c^{[2]} \mid T$. But then $\operatorname{supp}\left(T \cdot c^{[-2]}\right) \subseteq\{a, b\}$ and $c \in \pi\left(T \cdot c^{[-2]}\right)$, hence again $T \cdot c^{[-2]}=a^{[3]} \cdot b^{[3]}$, so that $R$ is empty, which is a contradiction.

\subsection{Diameter of the Cayley digraph}

Next we state a general inequality that can be obtained by a similar argument as the proof of Proposition 5.5. This result is not used here, but seems worthwhile to mention, as it involves the diameter of Cayley digraphs, which (in contrast with the large Davenport constant for a non-abelian group) has a rather extensive literature:

Proposition 5.6. Let $X$ be a set of generators of a finite group $G$ and $\mathrm{Cay}(G, X)$ the corresponding Cayley digraph. Then $\mathrm{D}(G) \geq \operatorname{diam}(\operatorname{Cay}(G, X))+1$.

Proof. Let $1=g_{0}, g_{1}, \ldots, g_{d} \in G$ be a sequence of vertices on a non-self-crossing path of maximal length in $\operatorname{Cay}(G, X)$, so that $d=\operatorname{diam}(\operatorname{Cay}(G, X))$. Now set $s_{i}=g_{i-1}^{-1} g_{i}$ for all $i=1, \ldots, d$ and consider the sequence $S=s_{1} \cdot \ldots \cdot s_{d} \cdot g_{d}^{-1}$. By construction $S$ is a product-one sequence and we claim that it is an atom in $\mathcal{B}\left(X \cup\left\{g_{d}^{-1}\right\}\right)$. For otherwise $S=A \cdot B$ for some product-one sequences $A, B$ where $g_{d}^{-1} \in A$, and then we can order the elements of $A$ in such a way that we obtain an equality $s_{i_{1}} \cdots s_{i_{r}} g_{d}^{-1}=1$ where $r<d$. But since $s_{i} \in X$ for all $i$, this means that we have in $\operatorname{Cay}(G, X)$ a path of length $r$ from 1 to $g_{d}$, a contradiction.

Problem 5.7. Is there any relation between $\max _{X \subseteq G}\{\operatorname{diam}(\operatorname{Cay}(G, X))\}$ and $\mathrm{d}(G)$ ? More concretely, based on a little computer experimentation we raise the following question: does the inequality $\mathrm{d}(G) \geq \operatorname{diam}(\operatorname{Cay}(G, X))$ hold in general? 


\section{Computing the Davenport constants}

We take over the following notations from Section 5.1; let $\mathcal{F}=\mathcal{F}(G)$ be the monoid of all sequences of elements in $G$, let $\mathcal{M} \subseteq \mathcal{F}$ be the set of product-one free sequences and $\mathcal{A} \subseteq \mathcal{F}$ the set of atoms, i.e. all product-one sequences which cannot be written as the product of two non-empty product-one subsequences. For any $k \geq 1$ let $\mathcal{F}_{k} \subseteq \mathcal{F}$ denote the set of all sequences of length $k$ and set $\mathcal{M}_{k}=\mathcal{M} \cap \mathcal{F}_{k}, \mathcal{A}_{k}=\mathcal{A} \cap \mathcal{F}_{k}$. Moreover, $\mathcal{M}_{0}=\left\{1_{\mathcal{F}}\right\}$ consists of the empty sequence.

We propose two algorithms: one for enumerating all product-one free sequences and another one for computing all the atoms and thereby establishing the values of $\mathrm{d}(G)$ and $\mathrm{D}(G)$, respectively. We have implemented these algorithms in the GAP computer algebra system (see [16]) and used them to compute and/or verify the small and large Davenport constants given in Section 2.

The algorithms construct the set $\mathcal{M}$ (respectively $\mathcal{A}$ ) successively, as a union of the sets $\mathcal{M}_{k}$ (respectively $\mathcal{A}_{k}$ ). During the construction of the sets $\mathcal{M}_{k}$ or $\mathcal{A}_{k}$ we are not testing all elements of $\mathcal{F}_{k}$ for being product-one free or atomic (as this task would be practically unfeasible), but we limit the scope of our search to a much smaller subset defined in what follows.

A sequence $T$ is called a splitting of a sequence $S$ if $T=S \cdot g^{[-1]} \cdot x \cdot y$ for some $g \in \operatorname{supp}(S)$ and $x, y \in G$ such that $x y=g$. We denote by $S \prec T$ the fact that $T$ is a splitting of $S$. For any subset $\mathcal{S} \subset \mathcal{F}(G)$ we set $\gamma(\mathcal{S}):=\{T \in$ $\mathcal{F}(G): S \prec T$ for some $S \in \mathcal{S}\}$.

Lemma 6.1. For any $k \geq 1$ we have $\mathcal{M}_{k+1} \subseteq \gamma\left(\mathcal{M}_{k}\right)$ and $\mathcal{A}_{k+1} \subseteq \gamma\left(\mathcal{A}_{k}\right)$.

Proof. If $T=g \cdot h \cdot T^{\prime}$ is a product-one free (respectively atomic) sequence of length $k+1 \geq 2$, then obviously the sequence $S=(g h) \cdot T^{\prime}$ has length $k$ and is product-one free (respectively atomic) too. Moreover, $S \prec T$.

Remark 6.2. Observe that by this lemma if $\mathcal{M}_{k}=\emptyset$ for some $k \geq 1$ then $\mathcal{M}_{l}=\emptyset$ for every $l>k$. So the smallest $k$ such that $\mathcal{M}_{k}=\emptyset$ equals $\mathrm{d}(G)+1$. Similarly the smallest $k$ such that $\mathcal{A}_{k}=\emptyset$ is equal to $\mathrm{D}(G)+1$.

Another idea used for reducing the complexity of our algorithms was to enumerate the elements of $\mathcal{M}_{k}$ and $\mathcal{A}_{k}$ only "up to similarity". Recall from Section 5.3 that two sequences $S$ and $T$ are said to be similar if there is an automorphism $\alpha \in \operatorname{Aut}(G)$ such that $S=\alpha(T)$.

Lemma 6.3. Let $\mathcal{R}_{k}$ be a set of $\operatorname{Aut}(G)$-orbit representatives over $\mathcal{M}_{k}$ (respectively $\left.\mathcal{A}_{k}\right)$. Then there is a system of $\operatorname{Aut}(G)$-orbit representatives $\mathcal{R}_{k+1}$ over $\mathcal{M}_{k+1}$ (respectively $\mathcal{A}_{k+1}$ ) such that $\mathcal{R}_{k+1} \subseteq \gamma\left(\mathcal{R}_{k}\right)$.

Proof. Observe that the relation $\prec$ is compatible with the $\operatorname{Aut}(G)$-action in the sense that $S \prec T$ holds if and only if $\alpha(S) \prec \alpha(T)$ for some $\alpha \in \operatorname{Aut}(G)$ if and only if $\alpha(S) \prec \alpha(T)$ for each $\alpha \in \operatorname{Aut}(G)$.

Now we prove that for any sequence $T \in \mathcal{M}_{k+1}$ its $\operatorname{Aut}(G)$-orbit $\operatorname{Orb}(T)$ has a non-empty intersection with $\gamma\left(\mathcal{R}_{k}\right)$. Take an arbitrary sequence $S \prec T$ so that $S \in \mathcal{M}_{k}$. As $\mathcal{R}_{k} \subseteq \mathcal{M}_{k}$ is a complete set of representatives, there is some $R \in \mathcal{R}_{k}$ such that $R=\alpha(S)$ for some $\alpha \in \operatorname{Aut}(G)$. Hence by the compatibility of $\prec$ it follows that $R=\alpha(S) \prec \alpha(T)$. As a result $\alpha(T) \in \operatorname{Orb}(T) \cap \gamma\left(\mathcal{R}_{k}\right)$ and we are done. The proof is similar for the case of $\mathcal{A}_{k}$. 


\subsection{Algorithm for computing the small Davenport con- stant}

Let us record the following obvious statement:

Lemma 6.4. A sequence $R$ of length $k \geq 1$ is product-one free if and only if $1 \notin \pi(R)$ and for all $g \in \operatorname{supp}(R)$ we have that $R \cdot g^{[-1]} \in \mathcal{M}_{k-1}$.

Given an arbitrary finite group $G$, Algorithm 1 computes the small Davenport constant. The set of all product-one free sequences of $G$ may be obtained as $\mathcal{M}=\bigcup_{k} \mathcal{M}_{k}$ after the repeat-until loop has terminated (right after line 33).

In lines 35 of the algorithm we have identified the sequences of length one with the respective group elements. Hence the set $\mathcal{M}_{1}$ of product-one free sequences having length one consists of all the group elements except the identity (line 3), from which we choose a set $\mathcal{R}_{1}$ of $\operatorname{Aut}(G)$-orbit representatives (line 4). For every length-one sequence we store the set of products (line 5). These initial data having been established, the algorithm proceeds with computing the sets $\mathcal{M}_{k}$ and $\mathcal{R}_{k}$ for all increasing $k>1$, until it finds the first value $k$ for which $\mathcal{M}_{k}=\emptyset$. The finiteness of the algorithm is guaranteed by Lemma 6.1 (together with Remark 6.2).

Observe that inside the for-loops in line 14 the variable $R$ will take successively all the values from the set $\gamma\left(\mathcal{R}_{k-1}\right)$, therefore it is guaranteed that for $k>1$ all the possible candidate sequences will be tested for being product-one free and the set $\mathcal{R}_{k}$ (from Lemma 6.3) is built correctly.

Before testing the sequence $R$ for being product-one free (using Lemma 6.4), in line 15] it is checked first whether the sequence $R$ is among the already computed product-one free sequences of length $k$ (via the condition $R \notin \mathcal{M}_{k}$ ). Elimination of duplicates is essential for correctly building the set $\mathcal{R}_{k}$ of representatives (so that in line 18 we compute only the set $\mathcal{R}_{k} \subseteq \gamma\left(\mathcal{R}_{k-1}\right)$ and not one of its supersets). If $R$ is a new sequence (in the sense that it is not similar to any of the already found product-one free sequences), it gets tested using the conditions from Lemma 6.4 (the second condition from line 15 and the condition from line 17). $\pi(R)$ is computed (in line 16) using the following observation: for any sequence $R$ and fixed $x \in R$, we can write

$$
\pi(R)=\bigcup_{R_{1} \cdot R_{2}=R \cdot x^{[-1]}} \pi\left(R_{1}\right) x \pi\left(R_{2}\right) .
$$

If $R$ is found to be product-one free, it is also added as a new representative of the Aut $(G)$-orbits (line 18). The orbit itself is computed successively (and stored in the variable $O_{R}$ ) by evaluating all the automorphisms $\alpha$ on the sequence $R$. In line 27 the set $O_{R}$ will be the same as the set $\operatorname{Orb}(R)$ of $\operatorname{Aut}(G)$ orbits of $R$.

For every new sequence $R^{\prime}$ (new in the sense of not being in the already computed part of the orbit), we also have to compute and store the set $\pi\left(R^{\prime}\right)$ by acting with the proper automorphism $\alpha$ on the set $\pi(R)$ (line 24). Hence the algorithm stores the product sets for all the enumerated product-one free sequences. This is essential for a relatively fast computation performed in line 16.

The set $\mathcal{M}_{k}$ gets computed in line 27 as a union of $\operatorname{Aut}(G)$ orbits of the representatives. In the last step, the value $k-1$ is returned: this is the small Davenport constant of $G$, as explained in Remark 6.2 


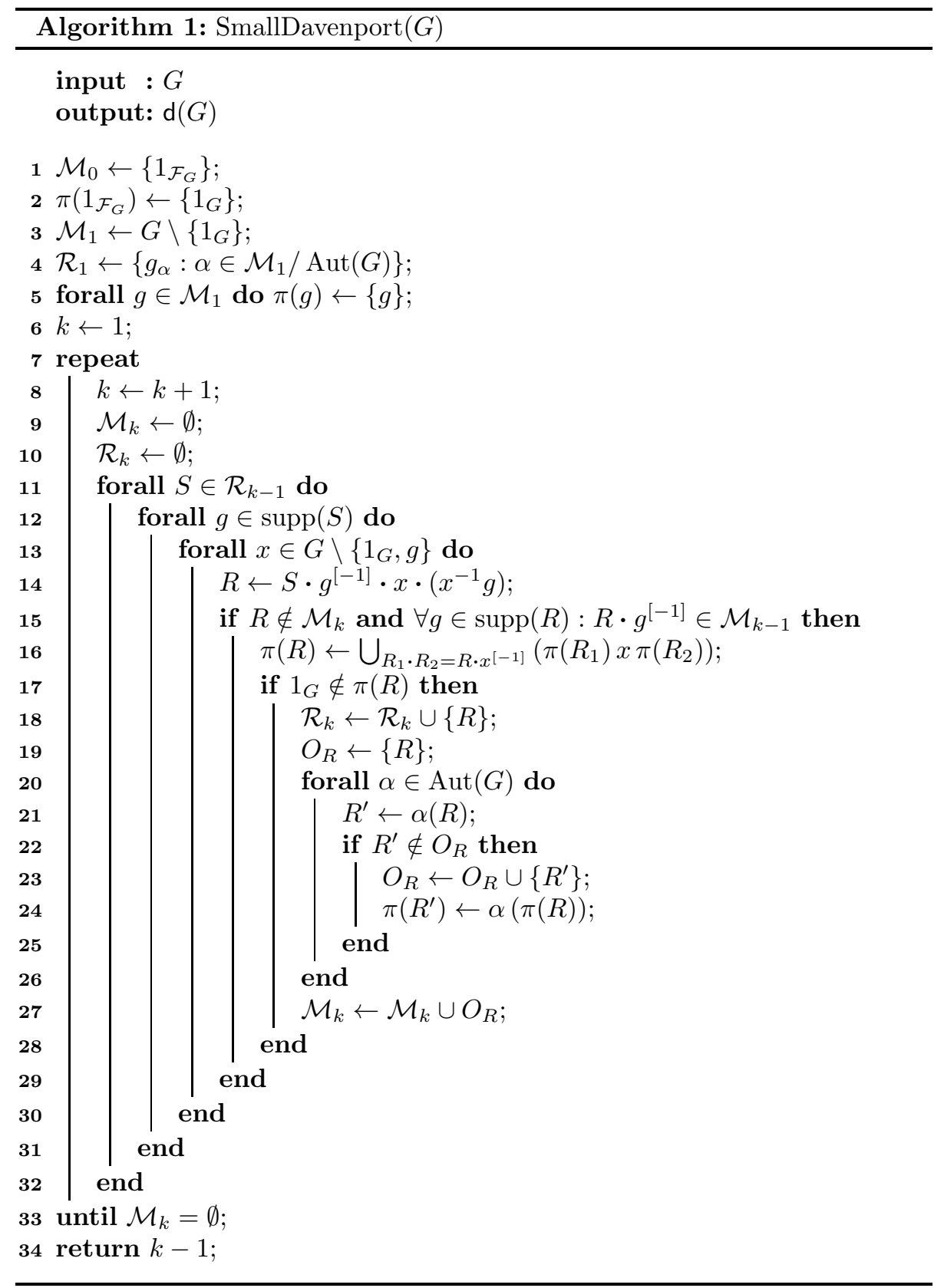




\subsection{Algorithm for computing the large Davenport con- stant}

Given an arbitrary finite group $G$, Algorithm 2 computes the large Davenport constant. The set of all atoms of $G$ may be obtained as $\mathcal{A}=\bigcup_{k} \mathcal{A}_{k}$ after the repeat-until loop has terminated (right after line 19).

The algorithm begins with loading the sets $\mathcal{A}_{1}$ and $\mathcal{R}_{1}$ with the sequence consisting only of the identity element of the group. In line 11 the variable $S^{\prime}$ will take successively all the values from the set $\gamma\left(\mathcal{R}_{k-1}\right)$, therefore it is guaranteed that for $k>1$ all the possible candidate sequences will be tested for being atoms and the set $\mathcal{A}_{k}$ (from Lemma 6.3) is built correctly. In line 10 the variable $h$ is prevented from taking the values $1_{G}$ or $g$, since this would bring in the identity element in the support of $S^{\prime}$.

The algorithm first checks whether a candidate sequence $S^{\prime}$ is among the already computed atoms of length $k$ via the condition $S^{\prime} \notin \mathcal{A}_{k}$ from line 12, ensuring that $S^{\prime}$ is a new atom (in the sense that it is not similar to any of the already found atoms of length $k$ ). Checking whether a sequence $S^{\prime}$ is an atom is performed by the second condition in line 12 by definition the product-one sequence $S^{\prime}$ is an atom if it cannot be written as a product of shorter atoms. The predicate $\mathbf{D}\left(S^{\prime}, k\right)$ in line 12 is true if and only if the product-one sequence $S^{\prime}$ of length $k$ is not an atom and may be given in the following way:

$$
\begin{aligned}
\mathbf{D}\left(S^{\prime}, k\right) \Longleftrightarrow & \exists A \in \bigcup_{i=2}^{\left\lfloor\frac{k}{2}\right\rfloor} \mathcal{A}_{i} \text { with } A \mid S^{\prime} \text { such that } \\
& S^{\prime} \cdot A^{[-1]} \in \mathcal{A}_{k-|A|} \text { or } \mathbf{D}\left(S^{\prime} \cdot A^{[-1]}, k-|A|\right) .
\end{aligned}
$$

It may be implemented using a recursive function, bailing out with a positive answer as soon as it finds a decomposition of $S^{\prime}$ into atoms.

In line 13 the newly found atom $S^{\prime}$ gets added to the set $\mathcal{R}_{k}$ of representatives and the set $\mathcal{A}_{k}$ of atoms is completed with the $\operatorname{Aut}(G)$ orbit of $S^{\prime}$, denoted by $\operatorname{Orb}\left(S^{\prime}\right)$ (in line 14). In the last step, the value $k-1$ is returned as the large Davenport constant of $G$, as explained in Remark 6.2

Although the check is performed only for a system of representatives of the $\operatorname{Aut}(G)$ orbits of atoms (for all $S^{\prime} \in \mathcal{R}_{k-1}$ ), the "decomposability test" described by the predicate $\mathbf{D}\left(S^{\prime}, k\right)$ uses the whole set of already computed atoms $\bigcup_{i=2}^{k-1} \mathcal{A}_{i}$, which is the main performance bottleneck of the algorithm. 


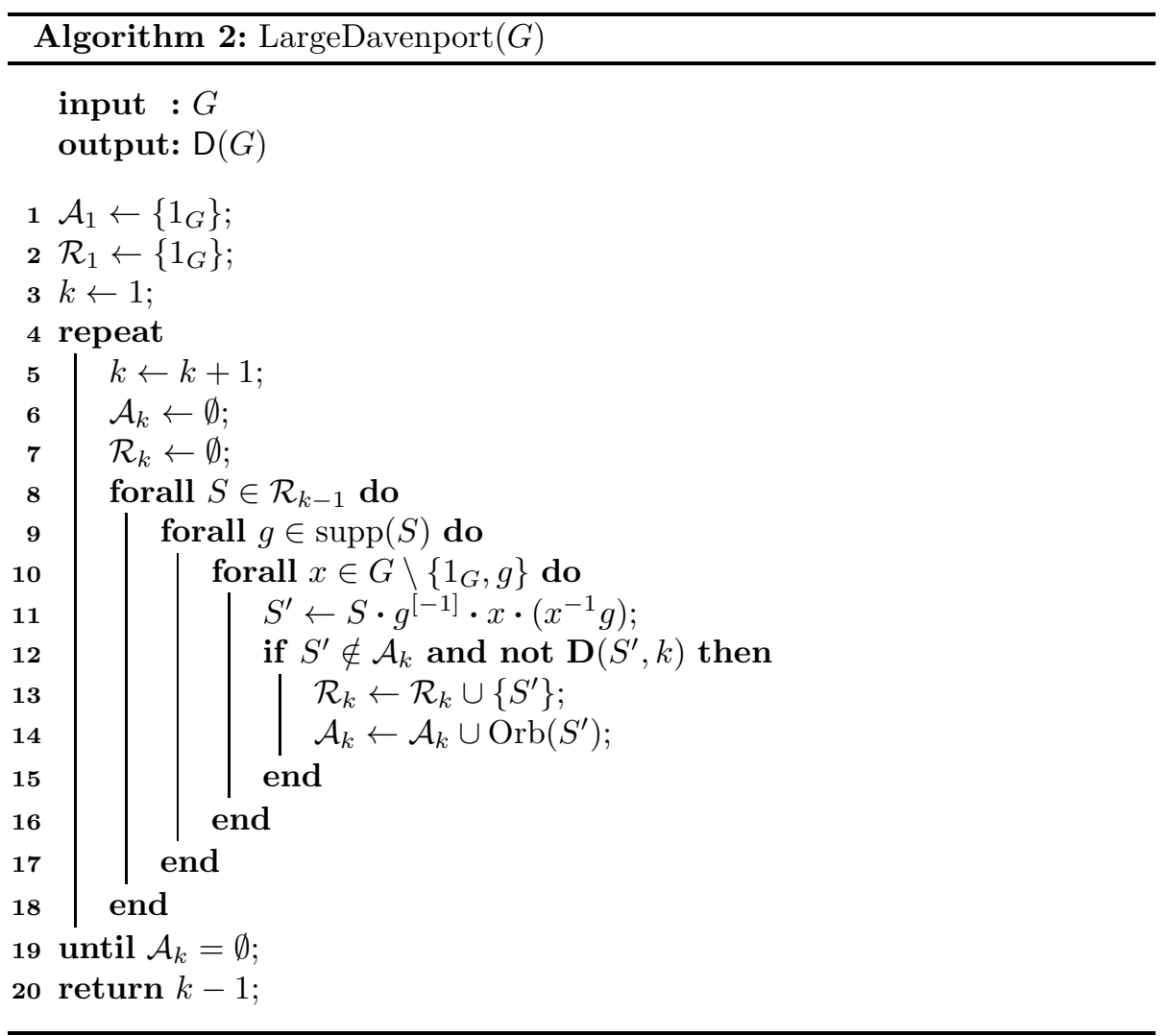

Remark 6.5. We have used a GAP implementation of Algorithm 1 and 2 to compute the small and large Davenport constants from the table in Section 2 in case of those groups for which a formula for these constants in not readily available (see Subsection 5.2 ).

Although parallelization is easily achievable in case of both algorithms, a "single-threaded" implementation was found to fit our present requirements, using a personal computer clocked at $2 \mathrm{GHz}$.

The small Davenport constants from Section 2 have been obtained in under a minute worth of computation, with the single exception of the group $M_{27}=C_{9} \rtimes$ $C_{3}$, for which $\mathrm{d}\left(M_{27}\right)=10$ has been obtained in about 7 minutes. This group was found to have a number of 102212 product-one free sequences, grouped in 1987 equivalence classes. In contrast, the second most "difficult" group (among the non-abelian groups of order less than 32 that contain no cyclic subgroup of index two) was $H_{27}=U T_{3}\left(\mathbb{F}_{3}\right)$ for which our implementation of Algorithm 1 yielded the following data in about 40 seconds: $\mathrm{d}\left(H_{27}\right)=6$ with 69026 productone free sequences partitioned by the similarity relation into 187 equivalence classes. In case of the other groups the running times range from below one second up to half a minute.

Computation of the large Davenport constant using Algorithm 2 is more demanding. The most time-consuming group from the list was $S L_{2}\left(\mathbb{F}_{3}\right)=\tilde{A}_{4}$, with the large Davenport constant being 13, housing 499695 atoms partitioned in 21033 equivalence classes. In the case of this group the computation took about 40 minutes. Excepting $C_{3} \rtimes D i h_{8}=\left(C_{6} \times C_{2}\right) \rtimes_{\gamma} C_{2}$ and $S_{4}$ (computation 
taking about 24 minutes for each of them), the running times hardly reached one minute. For example in the interesting case of $H_{27}$, the large Davenport constant $\mathrm{D}\left(\mathrm{H}_{27}\right)=8$ was computed in 19 seconds. This group has 108827 atoms grouped in only 340 equivalence classes (this explaining the relative quick computation within this group).

\section{References}

[1] G. Bhowmik and J.-C. Schlage-Puchta, Davenport's constant for groups of the form $\mathbb{Z}_{3} \oplus \mathbb{Z}_{3} \oplus \mathbb{Z}_{3 d}$, Additive combinatorics, 307-326, CRM Proc. Lecture Notes, 43, Amer. Math. Soc., Providence, RI, 2007.

[2] F. Chen and S. Savchev, Long minimal zero-sum sequences in the groups $C_{2}^{r-1} \oplus C_{2 k}$, Integers 14 (2014), Paper No. A 23, 29 pp.

[3] C. Chevalley, Invariants of finite groups generated by reflections, Amer. J. Math. 77 (1955), 778-782.

[4] K. Cziszter, The Noether number of the non-abelian group of order $3 p$, Periodica Math. Hungarica 68 (2014), 150-159.

[5] K. Cziszter, On the Noether number of $p$-groups, arXiv:1604.01938

[6] K. Cziszter and M. Domokos, On the generalized Davenport constant and the Noether number, Central European Journal of Mathematics 11 (2013), 1605-1615.

[7] K. Cziszter and M. Domokos, Groups with large Noether bound, Ann. Inst. Fourier (Grenoble) 64, no. 3 (2014), 909-944.

[8] K. Cziszter and M. Domokos, The Noether number for the groups with a cyclic subgroup of index two, Journal of Algebra 399 (2014), 546-560.

[9] K. Cziszter and M. Domokos, Lower bounds on the Noether number, Transform. Groups, 2018, DOI 10.1007/s00031-018-9479-4.

[10] K. Cziszter, M. Domokos, and A. Geroldinger, The interplay of invariant theory with multiplicative ideal theory and with arithmetic combinatorics, in: Scott T. Chapman, M. Fontana, A. Geroldinger, B.Olberding (Eds.), Multiplicative Ideal Theory and Factorization Theory, SpringerVerlag, 2016, pp. 43-95.

[11] Ch. Delorme, O. Ordaz, and D. Quiroz, Some remarks on Davenport constant, Discrete Mathematics 237 (2001),119-128.

[12] J. Draisma, G. Kemper, and D.Wehlau, Polarization of separating invariants, Canad. J. Math. Soc. 60 (2008), 556-571.

[13] P. van Emde Boas and D. Kruyswijk. A combinatorial problem on finite abelian groups. III. Math. Centrum Amsterdam Afd. Zuivere Wisk., 1969(ZW-008):32 pp., 1969.

[14] P. Fleischmann, The Noether bound in invariant theory of finite groups, Adv. Math. 156 (2000), 23-32. 
[15] J. Fogarty, On Noether's bound for polynomial invariants of a finite group, Electron. Res. Announc. Amer. Math. Soc. 7 (2001), 5-7.

[16] The GAP Group, GAP - Groups, Algorithms, and Programming, Version 4.8.6; 2016, (http://www.gap-system.org).

[17] A. Geroldinger, Additive group theory and non-unique factorizations, Combinatorial number theory and additive group theory, 1-86, Adv. Courses Math. CRM Barcelona, Birkhäuser Verlag, Basel, 2009.

[18] A. Geroldinger and D. J. Grynkiewicz, The large Davenport constant I: Groups with a cyclic index 2 subgroup, J. Pure Appl. Algebra 217 (2013), 863-885.

[19] A. Geroldinger and F. Halter-Koch, Non-Unique Factorizations. Algebraic, Combinatorial and Analytic Theory, Pure and Applied Mathematics, vol. 278, Chapman \& Hall/CRC, 2006.

[20] D. J. Grynkiewicz, The large Davenport constant II: General upper bounds, J. Pure Appl. Algebra 217 (2013), 222-2246.

[21] D. J. Grynkiewicz, Structural Additive Theory, Developments in Mathematics, Springer, 2013.

[22] F. Halter-Koch, A generalization of Davenport's constant and its arithmetical applications, Colloquium Mathematicum LXIII (1992), 203-210.

[23] M. Hunziker, Classical invariant theory for finite reflection groups, Transformation groups 2 (1997), 145-161.

[24] J.F. Humphreys, A course in group theory, Oxford University Press, 1996.

[25] F. Knop, On Noether's and Weyl's bound in positive characteristic, in "Invariant Theory in All Characteristics", (Ed.: H. E. A. Eddy Campbell and D. L. Wehlau), CRM Proceedings and Lecture Notes 35, Amer. Math. Soc., Providence, Rhode Island, pp. 175-188, 2004.

[26] E. Noether, Der Endlichkeitssatz der Invarianten endlicher Gruppen, Math. Ann. 77 (1916), 89-92.

[27] E. Noether, Der Endlichkeitssatz der Invarianten endlicher linearer Gruppen der Charakteristik $p$, Nachr. Ges. Wiss. Göttingen (1926), 28-35.

[28] J. E. Olson and E. T. White, Sums from a sequence of group elements, in: H. Zassenhaus (Ed.), Number Theory and Algebra, Academic Press, 1977, pp. 215-222.

[29] D. R. Richman, Invariants of finite groups over fields of characteristic $p$, Adv. Math. 124 (1996), 25-48.

[30] B. J. Schmid, Finite groups and invariant theory, in "Topics in invariant theory" (M.-P. Malliavin, ed.), Lecture notes in mathematics, no. 1478, Springer, 1989-90, pp. 35-66. 
[31] W. A. Schmid, The inverse problem associated to the Davenport constant for $C_{2} \oplus C_{2} \oplus C_{2 n}$, and applications to the arithmetical characterization of class groups, Electron. J. Combin. 18 (2011), no. 1, Paper 33, 42 pp.

[32] M. Sezer, Sharpening the generalized Noether bound in the invariant theory of finite groups, J. Algebra 254 (2002), 252-263.

[33] G. C. Shephard and J. A. Todd, Finite unitary reflection groups, Canad. J. Math. 6 (1954), 274-304.

[34] D. L. Wehlau, Some problems in invariant theory, in "Invariant Theory in All Characteristics", (Ed.: H. E. A. Eddy Campbell and D. L. Wehlau), CRM Proceedings and Lecture Notes 35, Amer. Math. Soc., Providence, Rhode Island, pp. 265-274, 2004.

[35] H. Weyl, The classical groups, Second edition, Princeton University Press, Princeton, 1946. 